\title{
Combining extensions of the Hales-Jewett Theorem with Ramsey Theory in other structures
}

\author{
Neil Hindman \\ Department of Mathematics \\ Howard University \\ Washington, DC, U.S.A. \\ nhindman@aol.com
}

\author{
Dona Strauss \\ Department of Pure Mathematics \\ University of Leeds \\ Leeds LS2 9J2, UK. \\ d.strauss@emeritus.hull.ac.uk
}

\author{
Luca Q. Zamboni \\ Université de Lyon \\ Université Lyon 1, CNRS UMR 5208 \\ Institut Camille Jordan \\ F69622 Villeurbanne Cedex, France. \\ zamboni@math.univ-lyon1.fr
}

Submitted: Jun 23, 2018; Accepted: Sep 30, 2019; Published: Oct 25, 2019

(C) The authors. Released under the CC BY-ND license (International 4.0).

\begin{abstract}
The Hales-Jewett Theorem states that given any finite nonempty set $\mathbb{A}$ and any finite coloring of the free semigroup $S$ over the alphabet $\mathbb{A}$ there is a variable word over $\mathbb{A}$ all of whose instances are the same color. This theorem has some extensions involving several distinct variables occurring in the variable word. We show that, when combined with a sufficiently well behaved homomorphism, the relevant variable word simultaneously satisfies a Ramsey-Theoretic conclusion in the other structure. As an example we show that if $\tau$ is the homomorphism from the set of variable words into the natural numbers which associates to each variable word $w$ the number of occurrences of the variable in $w$, then given any finite coloring of $S$ and any infinite sequence of natural numbers, there is a variable word $w$ whose instances are monochromatic and $\tau(w)$ is a sum of distinct members of the given sequence.

Our methods rely on the algebraic structure of the Stone-Čech compactification of $S$ and the other semigroups that we consider. We show for example that if $\tau$ is as in the paragraph above, there is a compact subsemigroup $P$ of $\beta \mathbb{N}$ which contains all of the idempotents of $\beta \mathbb{N}$ such that, given any $p \in P$, any $A \in p$, and any finite coloring of $S$, there is a variable word $w$ whose instances are monochromatic and $\tau(w) \in A$.
\end{abstract}


We end with a new short algebraic proof of an infinitary extension of the GrahamRothschild Parameter Sets Theorem.

Mathematics Subject Classifications: 05D10, 54D80

\section{Introduction}

We let $\mathbb{N}$ be the set of positive integers and let $\omega=\mathbb{N} \cup\{0\}$. Then $\omega$ is the first infinite ordinal.

Given a nonempty set $A$ (or alphabet) we let $A^{+}$be the set of all finite words $w=$ $a_{1} a_{2} \cdots a_{n}$ with $n \geqslant 1$ and $a_{i} \in A$. The quantity $n$ is called the length of $w$ and denoted $|w|$. The set $A^{+}$is naturally a semigroup under the operation of concatenation of words, known as the free semigroup over $A$. For each $u \in A^{+}$and $a \in A$, we let $|u|_{a}$ be the number of occurrences of $a$ in $u$. As is customary, we will identify the elements of $A$ with the length one words over $A$.

Throughout this paper we will let $\mathbb{A}$ be a nonempty set, let $S_{0}=\mathbb{A}^{+}$be the free semigroup over $\mathbb{A}$, and let $v$ (a variable) be a letter not belonging to $\mathbb{A}$. By a variable word over $\mathbb{A}$ we mean a word $w$ over $\mathbb{A} \cup\{v\}$ with $|w|_{v} \geqslant 1$. We let $S_{1}$ be the set of variable words over $\mathbb{A}$. If $w \in S_{1}$ and $a \in \mathbb{A}$, then $w(a) \in S_{0}$ is the result of replacing each occurrence of $v$ by $a$. For example if $\mathbb{A}=\{a, b, c\}$ and $w=a v b v v a$, then $w(a)=a a b a a a$ while $w(c)=$ acbcca. A finite coloring of a set $X$ is a function from $X$ to a finite set. A subset $A$ of $X$ is monochromatic if the function is constant on $A$.

Theorem 1 (A. Hales and R. Jewett). Assume that $\mathbb{A}$ is finite. For each finite coloring of $S_{0}$ there exists a variable word $w$ such that $\{w(a): a \in \mathbb{A}\}$ is monochromatic.

Proof. [5, Theorem 1].

Some extensions of the Hales-Jewett Theorem, including for example Theorem 3 or the Graham-Rothschild Parameter Sets Theorem [4] (see Theorem 4 below), involve the notion of $n$-variable words.

Definition 2. Let $n \in \mathbb{N}$ and $v_{1}, v_{2}, \ldots, v_{n}$ be distinct variables which are not members of $\mathbb{A}$.

(a) An $n$-variable word over $\mathbb{A}$ is a word $w$ over $\mathbb{A} \cup\left\{v_{1}, v_{2}, \ldots, v_{n}\right\}$ such that $|w|_{v_{i}} \geqslant 1$ for each $i \in\{1,2, \ldots, n\}$.

(b) If $w$ is an $n$-variable word over $\mathbb{A}$ and $\vec{x}=\left(x_{1}, x_{2}, \ldots, x_{n}\right)$, then $w(\vec{x})$ is the result of replacing each occurrence of $v_{i}$ in $w$ by $x_{i}$ for each $i \in\{1,2, \ldots, n\}$.

(c) If $w$ is an $n$-variable word over $\mathbb{A}$ and $u=l_{1} l_{2} \cdots l_{n}$ is a length $n$ word, then $w(u)$ is the result of replacing each occurrence of $v_{i}$ in $w$ by $l_{i}$ for each $i \in\{1,2, \ldots, n\}$.

(d) A strong $n$-variable word is an $n$-variable word such that for each $i \in\{1,2, \ldots, n-1\}$, the first occurrence of $v_{i}$ precedes the first occurrence of $v_{i+1}$. 
(e) $S_{n}$ is the set of $n$-variable words over $\mathbb{A}$ and $\widetilde{S}_{n}$ is the set of strong $n$-variable words over $\mathbb{A}$.

(f) $\widetilde{S}_{0}=S_{0}$.

(g) If $m \in \omega$ and $m<n$, then $\widetilde{S}\left(\begin{array}{c}n \\ m\end{array}\right)$ is the set of $u \in \widetilde{S}_{m}$ such that $|u|=n$.

(h) If $m \in \omega$ and $m<n$, then $S\left(\begin{array}{c}n \\ m\end{array}\right)$ is the set of $u \in S_{m}$ such that $|u|=n$.

The notation above does not reflect the dependence on the alphabet $\mathbb{A}$.

We note that if $m, n \in \omega$ and $m<n$, then for each $w \in \widetilde{S}_{n}$ and each $u \in \widetilde{S}\left(\begin{array}{c}n \\ m\end{array}\right)$, the word $w(u)$ belongs to $\widetilde{S}_{m}$.

The following is a first simple example of a multivariable extension of the Hales-Jewett Theorem:

Theorem 3. Assume that $\mathbb{A}$ is finite. Let $S_{0}$ be finitely colored and let $n \in \mathbb{N}$. There exists $w \in S_{n}$ such that $\left\{w(\vec{x}): \vec{x} \in \mathbb{A}^{n}\right\}$ is monochromatic.

Theorem 3 follows immediately from the Hales-Jewett Theorem applied to the alphabet $\mathbb{A}^{n}$, replacing each occurrence of $v$ in the variable word over $\mathbb{A}^{n}$ by $v_{1} v_{2} \cdots v_{n}$. It is also a consequence of Theorem 17, which constitutes one of the main results of this paper. (See the paragraph immediately following Theorem 49.) Theorem 3 also follows directly from Theorem 5 later in this section.

It is natural to ask the following question. Assume that $\mathbb{A}$ is finite. Let $S_{\infty}$ be the set of infinite words over $\mathbb{A} \cup\left\{v_{i}: i \in \mathbb{N}\right\}$ in which each $v_{i}$ occurs and assume that the set $\mathbb{A}^{\mathbb{N}}$ of infinite words over $\mathbb{A}$ is finitely colored. Must there exist $w \in S_{\infty}$ such that $\left\{w(\vec{x}): \vec{x} \in \mathbb{A}^{\mathbb{N}}\right\}$ is monochromatic, where $w(\vec{x})$ has the obvious meaning? As long as $|\mathbb{A}| \geqslant 2$, the answer is easily seen to be "no" already with 2-colorings, using a standard diagonalization argument: One has that $\left|\mathbb{A}^{\mathbb{N}}\right|=\left|S_{\infty}\right|=\mathfrak{c}$, so one may inductively color two elements of $\mathbb{A}^{\mathbb{N}}$ for each $w \in S_{\infty}$ so that there exist $\vec{x}$ and $\vec{y}$ in $\mathbb{A}^{\mathbb{N}}$ with the color of $w(\vec{x})$ and $w(\vec{y})$ different. (When one gets to $w$, fewer than $\mathfrak{c}$ things have been colored and there are $\mathfrak{c}$ distinct values of $w(\vec{x})$ possible.)

The following simplified version of the Graham-Rothschild Parameter Sets Theorem constitutes yet another fundamental multivariable extension of the Hales-Jewett Theorem. It was shown in [2, Theorem 5.1] that the full version as stated in [4, Section 4] can be easily derived from the version stated here.

Theorem 4 (R. Graham and B. Rothschild). Assume that $\mathbb{A}$ is finite. Let $m, n \in \omega$ with $m<n$ and let $\widetilde{S}_{m}$ be finitely colored. There exists $w \in \widetilde{S}_{n}$ such that $\left\{w(u): u \in \widetilde{S}\left(\begin{array}{c}n \\ m\end{array}\right)\right\}$ is monochromatic. 
After identifying the elements of $\mathbb{A}$ with the length 1 words over $\mathbb{A}$, one sees that Theorem 1 is exactly the $m=0$ and $n=1$ case of Theorem 4 . Notice also that Theorem 3 is actually equivalent to Theorem 4 in the special case of $m=0$. In fact if $w \in S_{n}, \sigma$ is a permutation of $\{1,2, \ldots, n\}$ and $u$ is the result of replacing each $v_{i}$ in $w$ by $v_{\sigma(i)}$ for each $i \in\{1,2, \ldots, n\}$, then $\left\{u(\vec{x}): \vec{x} \in \mathbb{A}^{n}\right\}=\left\{w(\vec{x}): \vec{x} \in \mathbb{A}^{n}\right\}$. In this paper we shall be mostly concerned with cases of Theorem 4 with $m=0$ and arbitrary $n \in \mathbb{N}$. (We are not concerned with $m>0$ because the natural versions of our main theorems are not valid for $m>0$. We shall discuss this point in Remark 25.) Accordingly, from this point on until Section 6 we will not be concerned with the order of occurrence of the variables.

In contrast to Theorem 3, the Graham-Rothschild Parameter Sets Theorem does not appear to be deducible directly from the Hales-Jewett Theorem; at least we know of no such proof.

Our main results in this paper deal with obtaining $n$-variable words satisfying the Hales-Jewett Theorem and simultaneously relating to Ramsey-Theoretic results in some relevant semigroup. The paper is organized as follows:

In Section 2 we present our main theorems relating $S_{n}$ with other structures. In Section 3 we determine precisely which homomorphisms from $S_{n}$ to $(\mathbb{N},+)$ satisfy the hypotheses of our main theorem of Section 2, namely Theorem 17.

The statements and proofs of the results in this paper use strongly the algebraic structure of the Stone-Čech compactification of a discrete semigroup. We now present a brief description of this structure. For more details or for any unfamiliar facts encountered in this paper, we refer the reader to [6, Part I]. All topological spaces considered herein are assumed to be Hausdorff.

Let $S$ be a semigroup. For each $s \in S, \rho_{s}: S \rightarrow S$ and $\lambda_{s}: S \rightarrow S$ are defined by $\rho_{s}(x)=x s$ and $\lambda_{s}(x)=s x$. If $S$ is also a topological space, $S$ is said to be right topological if the map $\rho_{s}$ is continuous for every $s \in S$. In this case, the set of elements $s \in S$ for which $\lambda_{s}$ is continuous, is called the topological center of $S$.

The assumption that $S$ is compact and right topological has powerful algebraic implications. $S$ has a smallest two sided ideal $K(S)$ which is the union of all of the minimal right ideals, as well as the union of all of the minimal left ideals. The intersection of any minimal left ideal and any minimal right ideal is a group. In particular, $S$ has idempotents. Any left ideal of $S$ contains a minimal left ideal of $S$, and any right ideal of $S$ contains a minimal right ideal of $S$. So the intersection of any left ideal of $S$ and any right ideal of $S$ contains an idempotent. An idempotent in $S$ is said to be minimal if it is in $K(S)$. This is equivalent to being minimal in the ordering of idempotents defined by $p \leqslant q$ if $p q=q p=p$. For any semigroup $T$, we let $E(T)$ be the set of idempotents in $T$. So $E(K(S))$ is the set of minimal idempotents in $S$. If $q$ is any idempotent in $S$, there is a minimal idempotent $p \in S$ for which $p \leqslant q$.

Given a discrete semigroup $(T, \cdot)$, let $\beta T=\{p: p$ is an ultrafilter on $T\}$. We identify the principal ultrafilter $e(x)=\{A \subseteq T: x \in A\}$ with the point $x \in T$ and thereby pretend that $T \subseteq \beta T$. A base for the topology of $\beta T$ consists of the clopen sets $\bar{A}$ for all $A \subseteq T$, where $\bar{A}=\{p \in \beta T: A \in p\}$. The operation - on $T$ extends to an operation on $\beta T$, also denoted by $\cdot$ making $(\beta T, \cdot)$ a right topological semigroup with $T$ contained 
in its topological center. So, given $p, q \in \beta T, p \cdot q=\lim _{s \rightarrow p} \lim _{t \rightarrow q} s \cdot t$, where $s$ and $t$ denote elements of $T$. If $A \subseteq T, A \in p \cdot q$ if and only if $\left\{x \in T: x^{-1} A \in q\right\} \in p$, where $x^{-1} A=\{y \in T: x \cdot y \in A\}$. If $(T,+)$ is a commutative discrete semigroup, we will use + for the semigroup operation on $\beta T$, even though $\beta T$ is likely to be far from commutative. In this case, we have that $A \in p+q$ if and only if $\{x \in T:-x+A \in q\} \in p$, where $-x+A=\{y \in T: x+y \in A\}$.

A set $D \subseteq T$ is piecewise syndetic if and only if $D \in p$ for some $p \in K(\beta T)$ and is central if and only $D \in p$ for some idempotent $p \in K(\beta T)$. We will also need the following equivalent characterization of piecewise syndetic sets: $D$ is piecewise syndetic if and only if there exists a finite subset $G$ of $T$ with the property that for every finite subset $F$ of $T$ there exists $x \in T$ such that $F x \subseteq \bigcup_{t \in G} t^{-1} D$. (See [6, Theorem 4.40].) Given a sequence $\left\langle x_{n}\right\rangle_{n=1}^{\infty}$ and $m \in \mathbb{N}$, we set $F P\left(\left\langle x_{n}\right\rangle_{n=m}^{\infty}\right)=\left\{\prod_{t \in F} x_{t}: F \in \mathcal{P}_{f}(\mathbb{N})\right.$ and $\left.\min F \geqslant m\right\}$, where $\mathcal{P}_{f}(\mathbb{N})$ is the set of finite nonempty subsets of $\mathbb{N}$ and the products are computed in increasing order of indices. Then $\bigcap_{m=1}^{\infty} \overline{F P\left(\left\langle x_{n}\right\rangle_{n=m}^{\infty}\right)}$ is a compact semigroup so there is an idempotent $p$ with $F P\left(\left\langle x_{n}\right\rangle_{n=m}^{\infty}\right) \in p$ for every $m$. (See [6, Lemma 5.11].) If $p$ is any idempotent in $\beta T$ and $A \in p$, then there is a sequence $\left\langle x_{n}\right\rangle_{n=1}^{\infty}$ in $T$ such that $F P\left(\left\langle x_{n}\right\rangle_{n=1}^{\infty}\right) \subseteq A$. If the operation is denoted by + , we write $F S\left(\left\langle x_{n}\right\rangle_{n=m}^{\infty}\right)=\left\{\sum_{t \in F} x_{t}\right.$ : $F \in \mathcal{P}_{f}(\mathbb{N})$ and $\left.\min F \geqslant m\right\}$.

If $\gamma$ is a function from the discrete semigroup $T$ to a compact space $C$, then $\gamma$ has a continuous extension from $\beta T$ to $C$, which we will also denote by $\gamma$. If $\gamma: T \rightarrow W$, where $W$ is discrete, we will view the continuous extension as taking $\beta T$ to $\beta W$, unless we state otherwise. If $\gamma: T \rightarrow C$ is a homomorphism from $T$ into a compact right topological semigroup $C$, with $\gamma[T]$ contained in the topological center of $C$, then the continuous extension $\gamma: \beta T \rightarrow C$ is a homomorphism by [6, Corollary 4.22].

As consequences of the results of Section 2 we establish that for $k \in \mathbb{N}$, the set of points $\left(p_{1}, p_{2}, \ldots, p_{k}\right) \in(\beta \mathbb{N})^{k}$ with the property that whenever $B_{i} \in p_{i}$ for $i \in\{1,2, \ldots, k\}$, the $k$-tuple $\left(B_{1}, B_{2}, \ldots, B_{k}\right)$ satisfies the conclusions of one of those theorems, is a compact subsemigroup of $(\beta \mathbb{N})^{k}$ containing the idempotents of $(\beta \mathbb{N})^{k}$ (or the minimal idempotents, depending on the theorem). The details of these results will be presented in Section 4 .

In Section 5 we restrict our attention to versions of the Hales-Jewett Theorem. Letting $R_{n}=\left\{p \in \beta S_{0}:(\forall B \in p)\left(\exists w \in S_{n}\right)\left(\left\{w(\vec{x}): \vec{x} \in \mathbb{A}^{n}\right\} \subseteq B\right)\right\}$, we show that each $R_{n}$ is a compact ideal of $\beta S_{0}$, that $R_{n+1} \subsetneq R_{n}$ for each $n \in \mathbb{N}$, and that $c \ell K\left(\beta S_{0}\right) \subsetneq \bigcap_{n=1}^{\infty} R_{n}$.

In Section 6 we present a new fully algebraic proof of an infinitary extension of the Graham-Rothschild Parameter Sets Theorem. This new proof is a significant simplification of the original.

We end this section with a few simple illustrations of how the algebraic structure described above may be applied to derive simple algebraic proofs of some of the results discussed earlier including for instance the Hales-Jewett Theorem. We begin with the following theorem whose proof is based on an argument due to Andreas Blass which first appeared in [1]. We regard this theorem as an algebraic extension of Theorem 3.

Theorem 5. Let $T$ be a semigroup and let $S$ be a subsemigroup of $T$. Let $F$ be a nonempty set of homomorphisms mapping $T$ to $S$ which are equal to the identity on $S$. 
(1) Let $p$ be a minimal idempotent in $\beta S$. Let $q$ be an idempotent in $\beta T$ for which $q \leqslant p$. Then $\nu(q)=p$ for every $\nu \in F$.

(2) For any finite subset $F_{0}$ of $F$ and any central subset $D$ of $S$, there is a central subset $Q$ of $T$ such that, for every $t \in Q,\left\{\nu(t): \nu \in F_{0}\right\} \subseteq D$.

(3) For any finite subset $F_{0}$ of $F$ and any finite coloring of $S$, there is a central subset $Q$ of $T$ such that, for every $t \in Q,\left\{\nu(t): \nu \in F_{0}\right\}$ is monochromatic.

Proof. (1) For each $\nu \in F, \nu(q) \leqslant \nu(p)=p$ and so, since $\nu(q) \in \beta S, \nu(q)=p$.

(2) Pick a minimal idempotent $p \in \beta S$ such that $D \in p$. By [6, Theorem 1.60], pick a minimal idempotent $q \in \beta T$ such that $q \leqslant p$. Then $\nu(q)=p$ for every $\nu \in F_{0}$. Hence, if $Q=\bigcap_{\nu \in F_{0}} \nu^{-1}[D]$, then $Q \in q$.

(3) Pick a minimal idempotent $p \in \beta S$ and let $D$ be a monochromatic member of $p$.

We note that the above theorem provides an algebraic proof of Theorem 3 and hence of the Hales-Jewett Theorem. In fact, put $S=S_{0}, T=S_{0} \cup S_{n}$ and $F=\left\{h_{\vec{x}}: \vec{x} \in \mathbb{A}^{n}\right\}$, where $h_{\vec{x}}(w)=\left\{\begin{array}{cl}w(\vec{x}) & \text { if } w \in S_{n} \\ w & \text { if } w \in S_{0}\end{array}\right.$. Then by Theorem 5 we deduce that for any finite coloring of $S_{0}$ there exists a central subset $Q$ of $T$ such that for every $w \in Q,\left\{w(\vec{x}): \vec{x} \in \mathbb{A}^{n}\right\}$ is monochromatic. Pick $q \in K(\beta T)$ with $Q \in q$. Then since $S_{n}$ is an ideal of $T$ it follows that $S_{n} \in q$. So for any $w \in S_{n} \cap Q$ we have $\left\{w(\vec{x}): \vec{x} \in \mathbb{A}^{n}\right\}$ is monochromatic.

We conclude this section with two additional simple corollaries of Theorem 5 that will not be needed in the rest of the paper.

Corollary 6. Let $T$ be a semigroup and let $S$ be a subsemigroup of $T$. Let $F$ be a finite nonempty set of homomorphisms mapping $T$ to $S$ which are equal to the identity on $S$. Let $D$ be a piecewise syndetic subset of $S$. Then $\bigcap_{\nu \in F} \nu^{-1}[D]$ is a piecewise syndetic subset of $T$.

Proof. By [6, Theorem 4.43], we may pick $s \in S$ for which $s^{-1} D$ is a central subset of $S$. We can choose a minimal idempotent $p$ in $\beta S$ for which $s^{-1} D \in p$, and we can then choose a minimal idempotent $q$ in $\beta T$ for which $q \leqslant p$, by [6, Theorem 1.60]. By Theorem $5(1), \nu(q)=p$ for every $\nu \in F$. Hence, if $Q=\bigcap_{\nu \in F} \nu^{-1}\left[s^{-1} D\right]$, then $Q \in q$. Now $s Q$ is a piecewise syndetic subset of $T$, because $s Q \in s q$ and $s q \in K(\beta T)$. We claim that $s Q \subseteq \bigcap_{\nu \in F} \nu^{-1}[D]$. In fact, let $x \in s Q$, pick $t \in Q$ such that $x=s t$, and let $\nu \in F$. Then $\nu(x)=\nu(s t)=s \nu(t) \in s\left(s^{-1} D\right) \subseteq D$.

Given an idempotent $p$ and $B \in p$ let $B^{\star}(p)=\left\{x \in B: x^{-1} B \in p\right\}$. Then $B^{\star}(p) \in p$ and for each $x \in B^{\star}(p)$, one has that $x^{-1} B^{\star}(p) \in p$. (See [6, Lemma 4.14]). If there is no risk of confusion, we will sometimes write $B^{\star}$ for $B^{\star}(p)$.

Corollary 7. Let $T$ be a semigroup and let $S$ be a subsemigroup of $T$. Let $F$ be a finite nonempty set of homomorphisms from $T$ onto $S$ which are equal to the identity on $S$. Let $p$ be a minimal idempotent in $\beta S$ and let $P \in p$. Let $q$ be a minimal idempotent of $\beta T$ for which $q \leqslant p$ and let $Q=\bigcap_{\nu \in F} \nu^{-1}\left[P^{\star}\right]$. Then $Q \in q$. There is an infinite 
sequence $\left\langle w_{n}\right\rangle_{n=1}^{\infty}$ of elements of $Q$ such that for each $H \in \mathcal{P}_{f}(\mathbb{N})$ and each $\varphi: H \rightarrow F$, $\prod_{t \in H} \varphi(t)\left(w_{t}\right) \in P^{\star}$, where the product is computed in increasing order of indices.

Proof. Choose $w_{1} \in Q$. Let $m \in \mathbb{N}$ and assume we have chosen $\left\langle w_{t}\right\rangle_{t=1}^{m}$ in $Q$ such that whenever $\varnothing \neq H \subseteq\{1,2, \ldots, m\}$ and $\varphi: H \rightarrow F, \prod_{t \in H} \varphi(t)\left(w_{t}\right) \in P^{\star}$. Note that this hypothesis is satisfied for $m=1$. Let

$$
E=\left\{\prod_{t \in H} \varphi(t)\left(w_{t}\right): \varnothing \neq H \subseteq\{1,2, \ldots, m\} \text { and } \varphi: H \rightarrow F\right\} .
$$

Then $E \subseteq P^{\star}$. Let $R=P^{\star} \cap \bigcap_{y \in E} y^{-1} P^{\star}$. Then $R \in p$ so $\bigcap_{\nu \in F} \nu^{-1}[R] \in q$. Pick $w_{m+1} \in \bigcap_{\nu \in F} \nu^{-1}[R]$ and note that $w_{m+1} \in Q$.

To verify the hypothesis let $\varnothing \neq H \subseteq\{1,2, \ldots, m+1\}$ and let $\varphi: H \rightarrow F$. If $m+1 \notin H$, the conclusion holds by assumption, so assume that $m+1 \in H$. If $H=\{m+1\}$, then $w_{m+1} \in \varphi(m+1)^{-1}\left[P^{\star}\right]$, so assume that $\{m+1\} \subsetneq H$ and let $G=H \backslash\{m+1\}$. Let $y=\prod_{t \in G} \varphi(t)\left(w_{t}\right)$. Then $w_{m+1} \in \varphi(m+1)^{-1}\left[y^{-1} P^{\star}\right]$ so $\prod_{t \in H} \varphi(t)\left(w_{t}\right)=y \varphi(m+$ 1) $\left(w_{m+1}\right) \in P^{\star}$.

The authors thank the referee for a thorough report.

\section{Combining structures}

Throughout this section, and up until Section $6, \mathbb{A}$ is a fixed non-empty finite alphabet. Most of the results in this paper involve families of well behaved homomorphisms between certain semigroups:

Definition 8. Let $n \in \mathbb{N}$ and let $\nu: S_{n} \rightarrow S_{0}$ be a homomorphism. We shall say that $\nu$ is $S_{0}$-preserving if $\nu(u w)=u \nu(w)$ and $\nu(w u)=\nu(w) u$ for every $u \in S_{0}$ and every $w \in S_{n}$.

Note that if $\vec{x} \in \mathbb{A}^{n}$, then the function $h_{\vec{x}}: S_{n} \rightarrow S_{0}$ defined by $h_{\vec{x}}(w)=w(\vec{x})$ is an $S_{0}$-preserving homomorphism. Also, the function $\delta: S_{n} \rightarrow S_{0}$ which simply deletes all occurrences of variables is an $S_{0}$-preserving homomorphism. As another example, assume that $n \geqslant 2$ and define $\mu: S_{n} \rightarrow S_{n}$ where $\mu(w)$ is obtained from $w$ by replacing each occurrence of $v_{2}$ by $v_{1} v_{2}$. Given $\vec{x} \in \mathbb{A}^{n}, h_{\vec{x}} \circ \mu$ is an $S_{0}$-preserving homomorphism which cannot be obtained by composing those of the kind mentioned previously; in fact $\left|h_{\vec{x}} \circ \mu(w)\right|>|w|$ for each $w \in S_{n}$.

Definition 9. Let $S, T$, and $R$ be semigroups such that $S \cup T$ is a semigroup and $T$ is an ideal of $S \cup T$. Then a homomorphism $\tau: T \rightarrow R$ is said to be $S$-independent if, for every $w \in T$ and every $u \in S, \tau(u w)=\tau(w)=\tau(w u)$.

In most cases, the above definition will be applied to the case $S=S_{0}$ and $T=S_{n}$ for some $n \in \mathbb{N}$. We shall see later in Lemma 28 that if $n \in \mathbb{N}, R$ is a cancellative commutative semigroup, and $\tau: S_{n} \rightarrow R$ is an $S_{0}$-independent homomorphism, then $\tau(w)=\tau\left(w^{\prime}\right)$ whenever $|w|_{v_{i}}=\left|w^{\prime}\right|_{v_{i}}$ for each $i \in\{1,2, \ldots, n\}$. For reasons which will be made clear in Section 3 , we will primarily be concerned with $S_{0}$-independent homomorphisms from $S_{n}$ to $(\mathbb{N},+)$ of the form $\tau(w)=|w|_{v_{i}}$ for some $i \in\{1,2, \ldots, n\}$. 
Lemma 10. Let $S$ and $T$ be semigroups such that $S \cup T$ is a semigroup and $T$ is an ideal of $S \cup T$. Let $\phi: T \rightarrow C$ be an $S$-independent homomorphism from $T$ into the topological center of a compact right topological semigroup $C$. Then $\phi$ extends to a continuous homomorphism from $\beta T$ into $C$, which we shall also denote by $\phi$. For every $q \in \beta T$ and every $p \in \beta S, \phi(q)=\phi(p q)=\phi(q p)$.

Proof. The fact that $\phi$ extends to a continuous homomorphism is [6, Corollary 4.22]. Let $p \in \beta S$ and $q \in \beta T$ be given. In the following expressions let $s$ and $t$ denote members of $S$ and $T$ respectively. Since $\phi$ is continuous on $\beta T$ and since both $p q$ and $q p$ are in $\beta T$ by [6, Corollary 4.18], we have that

$$
\phi(p q)=\phi\left(\lim _{s \rightarrow p} \lim _{t \rightarrow q} s t\right)=\lim _{s \rightarrow p} \lim _{t \rightarrow q} \phi(s t)=\lim _{t \rightarrow q} \phi(t)=\phi(q)
$$

and similarly

$$
\phi(q p)=\phi\left(\lim _{t \rightarrow q} \lim _{s \rightarrow p} t s\right)=\lim _{t \rightarrow q} \lim _{s \rightarrow p} \phi(t s)=\lim _{t \rightarrow q} \phi(t)=\phi(q) .
$$

Theorem 11. Let $S$ and $T$ be semigroups such that $S \cup T$ is a semigroup and $T$ is an ideal of $S \cup T$. Let $\phi: T \rightarrow C$ be an $S$-independent homomorphism from $T$ into a compact right topological semigroup $C$ with $\phi[T]$ contained in the topological center of $C$ and denote also by $\phi$ its continuous extension to $\beta T$. Let $F$ be a finite nonempty set of homomorphisms from $S \cup T$ into $S$ which are each equal to the identity on $S$, and let $D$ be a piecewise syndetic subset of $S$. Let $p$ be an idempotent in $\phi[\beta T]$, and let $U$ be a neighborhood of $p$ in $C$. There exists $w \in T$ such that $\phi(w) \in U$ and $\nu(w) \in D$ for every $\nu \in F$.

Proof. Since $D$ is piecewise syndetic in $S$, pick by [6, Theorem 4.43] some $s \in S$ such that $s^{-1} D$ is central in $S$ and pick a minimal idempotent $r \in \beta S$ such that $s^{-1} D \in r$.

Let $V=\phi^{-1}[\{p\}]$. Since $\phi$ is a continuous homomorphism from $\beta T$ to $C, V$ is a compact subsemigroup of $\beta T$. By Lemma 10, $V r$ is a left ideal of $V$ and $r V$ is a right ideal of $V$. Pick an idempotent $q \in V r \cap r V$ and note that $q \leqslant r$ in $\beta T$. By Theorem $5(1), \nu(q)=r$ for every $\nu \in F$.

Since $s^{-1} D \in r$ we have that for each $\nu \in F, \nu^{-1}\left[s^{-1} D\right] \in q$. Since $U$ is a neighborhood of $p$, pick $R \in q$ such that $\phi[\bar{R}] \subseteq U$. Pick $w \in R \cap \bigcap_{\nu \in F} \nu^{-1}\left[s^{-1} D\right]$. Then $\phi(s w)=$ $\phi(w) \in U$ and for $\nu \in F, \nu(w) \in s^{-1} D$ so $\nu(s w)=s \nu(w) \in D$.

Corollary 12. Let $n \in \mathbb{N}$. Let $\phi: S_{n} \rightarrow C$ be an $S_{0}$-independent homomorphism from $S_{n}$ into a compact right topological semigroup $C$ with $\phi\left[S_{n}\right]$ contained in the topological center of $C$ and denote also by $\phi$ the continuous extension to $\beta S_{n}$. Let $F$ be a finite nonempty set of $S_{0}$-preserving homomorphisms from $S_{n}$ into $S_{0}$, let $D$ be a piecewise syndetic subset of $S_{0}$, let $p$ be an idempotent in $\phi\left[\beta S_{n}\right]$, and let $U$ be a neighborhood of $p$ in $C$. There exists $w \in S_{n}$ such that $\phi(w) \in U$ and $\nu(w) \in D$ for every $\nu \in F$.

Proof. Let $S=S_{0}$, let $T=S_{n}$, and for $\nu \in F$, extend $\nu$ to $S_{0} \cup S_{n}$ by defining $\nu$ to be the identity on $S_{0}$. Then Theorem 11 applies.

We obtain the first result that was stated in the abstract as a corollary to Theorem 11. 
Corollary 13. Define $\tau: S_{1} \rightarrow \mathbb{N}$ by $\tau(w)=|w|_{v_{1}}$, let $S_{0}$ be finitely colored, and let $\left\langle x_{n}\right\rangle_{n=1}^{\infty}$ be a sequence in $\mathbb{N}$. There exists $w \in S_{1}$ such that $\{w(a): a \in \mathbb{A}\}$ is monochromatic and $\tau(w) \in F S\left(\left\langle x_{n}\right\rangle_{n=1}^{\infty}\right)$.

Proof. Let $S=S_{0}$, let $T=S_{1}$, and let $C=\beta \mathbb{N}$. Then $\tau\left[S_{1}\right]$ is contained in the topological center of $C$. Denote also by $\tau$ the continuous extension taking $\beta S_{1}$ to $\beta \mathbb{N}$. Given $a \in \mathbb{A}$, define $f_{a}: S_{0} \cup S_{1} \rightarrow S_{0}$ by

$$
f_{a}(w)=\left\{\begin{array}{cl}
w(a) & \text { if } w \in S_{1} \\
w & \text { if } s \in S_{0}
\end{array}\right.
$$

and let $F=\left\{f_{a}: a \in \mathbb{A}\right\}$. Then $F$ is a finite nonempty set of homomorphisms from $S_{0} \cup S_{1}$ into $S_{0}$ which are each equal to the identity on $S_{0}$. Pick by [6, Lemma 5.11] an idempotent $p \in \beta \mathbb{N}$ such that $F S\left(\left\langle x_{n}\right\rangle_{n=1}^{\infty}\right) \in p$. Pick any $q \in K\left(\beta S_{0}\right)$ and pick $D \in q$ which is monochromatic. Note that $\tau\left[S_{1}\right]=\mathbb{N}$ so by [6, Exercise 3.4.1], $\tau\left[\beta S_{1}\right]=\beta \mathbb{N}$. Therefore, $p \in \tau\left[\beta S_{1}\right]$. Consequently, Theorem 11 applies with $U=\overline{F S\left(\left\langle x_{n}\right\rangle_{n=1}^{\infty}\right)}$.

Lemma 14. Let $(T, \cdot)$ be a discrete semigroup and let $m, n \in \mathbb{N}$. Let $\phi: S_{n} \rightarrow \times_{i=1}^{m} T$ be an $S_{0}$-independent homomorphism. Then $\phi$ extends to a continuous $S_{0}$-independent homomorphism $\phi: \beta S_{n} \rightarrow \times_{i=1}^{m} \beta T$. Moreover if $\vec{p}=\left(p_{1}, p_{2}, \ldots, p_{m}\right)$ is an idempotent in $\times_{i=1}^{m} \beta T$ with the property that whenever $B_{i} \in p_{i}$ for each $i \in\{1,2, \ldots, m\}$, there exists $w \in S_{n}$ such that $\phi(w) \in \times_{i=1}^{m} B_{i}$, then $\vec{p} \in \phi\left[\beta S_{n}\right]$.

Proof. Let $C=\times_{i=1}^{m} \beta T$. Regarding $\phi$ as an $S_{0}$-independent homomorphism from $S_{n}$ into the right topological semigroup $C$, we see that $\phi\left[S_{n}\right]$ is contained in $\times_{i=1}^{m} T$ which in turn is contained in the topological center of $C$ by [6, Theorem 2.22]. Hence by [6, Corollary 4.22], $\phi$ extends to a continuous homomorphism from $\beta S_{n}$ into $C$. To see that the extension is $S_{0}$-independent, let $u \in S_{0}$ and let $p \in \beta S_{n}$. Then, letting $s$ denote a member of $S_{n}$, we have

$$
\phi(u p)=\phi\left(\lim _{s \rightarrow p} u s\right)=\lim _{s \rightarrow p} \phi(u s)=\lim _{s \rightarrow p} \phi(s)=\phi\left(\lim _{s \rightarrow p} s\right)=\phi(p)
$$

and similarly, $\phi(p u)=\phi(p)$.

Now assume that $\vec{p}=\left(p_{1}, p_{2}, \ldots, p_{m}\right)$ is an idempotent in $\times_{i=1}^{m} \beta T$ and whenever $B_{i} \in p_{i}$ for each $i \in\{1,2, \ldots, m\}$, there exists $w \in S_{n}$ such that $\phi(w) \in \times_{i=1}^{m} B_{i}$, To see that $\vec{p} \in \phi\left[\beta S_{n}\right]$ let $\left(B_{1}, B_{2}, \ldots, B_{m}\right) \in \times_{i=1}^{m} p_{i}$, and let

$$
G_{\left(B_{1}, \ldots, B_{m}\right)}=\left\{w \in S_{n}: \phi(w) \in \times_{i=1}^{m} B_{i}\right\} .
$$

Then by assumption, $\mathcal{G}=\left\{G_{\left(B_{1}, \ldots, B_{m}\right)}:\left(B_{1}, B_{2}, \ldots, B_{m}\right) \in \times_{i=1}^{m} p_{i}\right\}$ has the finite intersection property so one may pick $q \in \beta S_{n}$ such that $\mathcal{G} \subseteq q$. Then $\vec{p}=\phi(q) \in \phi\left[\beta S_{n}\right]$.

Theorem 15. Let $(T, \cdot)$ be a discrete semigroup and let $m, n \in \mathbb{N}$. Let $\vec{p}=\left(p_{1}, p_{2}, \ldots, p_{m}\right)$ be an idempotent in $\times_{i=1}^{m} \beta T$. For $i \in\{1,2, \ldots, m\}$ let $\tau_{i}$ be an $S_{0}$-independent homomorphism from $S_{n}$ to $T$. Assume that whenever $B_{i} \in p_{i}$ for each $i \in\{1,2, \ldots, m\}$, there exists $w \in S_{n}$ such that $\left(\tau_{1}(w), \tau_{2}(w), \ldots, \tau_{m}(w)\right) \in \times_{i=1}^{m} B_{i}$. Let $D$ be a piecewise syndetic subset of $S_{0}$ and let $F$ be a finite nonempty set of $S_{0}$-preserving homomorphisms from $S_{n}$ to $S_{0}$. Then whenever $B_{i} \in p_{i}$ for each $i \in\{1,2, \ldots, m\}$, there exists $w \in S_{n}$ such that $\nu(w) \in D$ for each $\nu \in F$ and for each $i \in\{1,2, \ldots, m\}, \tau_{i}(w) \in B_{i}$. 
Proof. Define $\phi: S_{n} \rightarrow \times_{i=1}^{m} T$ by

$$
\phi(w)=\left(\tau_{1}(w), \tau_{2}(w), \ldots, \tau_{m}(w)\right) .
$$

Then $\phi$ is an $S_{0}$-independent homomorphism and hence by Lemma $14, \phi$ extends to a continuous $S_{0}$-independent homomorphism $\phi: \beta S_{n} \rightarrow \times_{i=1}^{m} \beta T$ and $\vec{p} \in \phi\left[\beta S_{n}\right]$. The result now follows from Corollary 12.

Corollary 16. Let $k, n \in \mathbb{N}$ with $k<n$ and let $T$ be the set of words over $\left\{v_{1}, v_{2}, \ldots, v_{k}\right\}$ in which $v_{i}$ occurs for each $i \in\{1,2, \ldots, k\}$. Given $w \in S_{n}$ let $\tau(w)$ be obtained from $w$ by deleting all occurrences of elements of $\mathbb{A}$ as well as all occurrences of $v_{i}$ for $k<i \leqslant n$. Let $\left\langle y_{t}\right\rangle_{t=1}^{\infty}$ be a sequence in $T$, let $F$ be a finite nonempty set of $S_{0}$-preserving homomorphisms from $S_{n}$ to $S_{0}$, and let $D$ be a piecewise syndetic subset of $S_{0}$. There exists $w \in S_{n}$ such that $\nu(w) \in D$ for all $\nu \in F$ and $\tau(w) \in F P\left(\left\langle y_{t}\right\rangle_{t=1}^{\infty}\right)$.

Proof. Pick an idempotent $p \in \beta T$ such that $F P\left(\left\langle y_{t}\right\rangle_{t=1}^{\infty}\right) \in p$. Since $\tau$ is an $S_{0^{-}}$ independent homomorphism from $S_{n}$ onto $T$, Theorem 15 applies with $m=1$.

Theorem 17 is one of the main results of this paper. It involves a matrix with entries from $\mathbb{Q}$ or $\mathbb{Z}$. We assume appropriate hypotheses in order to ensure that In order to ensure that matrix multiplication makes sense and is distributive. In particular we assume that $T$ is commutative and write the operation as + .

Theorem 17. Let $(T,+)$ be a commutative semigroup, let $k, m, n \in \mathbb{N}$, and let $M$ be a $k \times m$ matrix. If $T$ is not cancellative assume that the entries of $M$ come from $\omega$. If $T$ is isomorphic to a subsemigroup of a direct sum of copies of $(\mathbb{Q},+$ ) (so that multiplication by members of $\mathbb{Q}$ makes sense), assume that the entries of $M$ come from $\mathbb{Q}$. Otherwise assume that the entries of $M$ come from $\mathbb{Z}$. For $i \in\{1,2, \ldots, m\}$ let $\tau_{i}$ be an $S_{0}$-independent homomorphism from $S_{n}$ to $T$. Define a function $\psi$ on $S_{n}$ by $\psi(w)=\left(\begin{array}{c}\tau_{1}(w) \\ \tau_{2}(w) \\ \vdots \\ \tau_{m}(w)\end{array}\right)$. Let $\vec{p}=\left(p_{1}, p_{2}, \ldots, p_{k}\right)$ be an idempotent in $\times_{i=1}^{k} \beta T$ with the property that whenever $B_{i} \in p_{i}$ for each $i \in\{1,2, \ldots, k\}$, there exists $\vec{z} \in \psi\left[S_{n}\right]$ such that $M \vec{z} \in \times_{i=1}^{k} B_{i}$. Let $F$ be a finite nonempty set of $S_{0}$-preserving homomorphisms from $S_{n}$ to $S_{0}$ and let $D$ be a piecewise syndetic subset of $S_{0}$. Then whenever $B_{i} \in p_{i}$ for each $i \in\{1,2, \ldots, k\}$, there exists $w \in S_{n}$ such that $\nu(w) \in D$ for every $\nu \in F$ and $M \psi(w) \in \times_{i=1}^{k} B_{i}$.

Proof. If $T$ is not cancellative, let $G=T$. If $T$ is isomorphic to a subsemigroup of $\bigoplus_{i \in I} \mathbb{Q}$ for some set $I$, assume without loss of generality that $T \subseteq \bigoplus_{i \in I} \mathbb{Q}$ and let $G=\bigoplus_{i \in I} \mathbb{Q}$. Otherwise let $G$ be the group of differences of $T$. In each case we define an $S_{0}$-independent homomorphism $\phi: S_{n} \rightarrow \times_{j=1}^{k} G$. by $\phi(w)=M \psi(w)$. Let $C=\times_{j=1}^{k} \beta G$. Then by Lemma 14, $\phi$ extends to an $S_{0}$-independent homomorphism $\phi: \beta S_{n} \rightarrow C$ and $\vec{p} \in \phi\left[\beta S_{n}\right]$. The rest now follows from Corollary 12 .

Definition 18. Let $n \in \mathbb{N}$ and let $j \in\{1,2, \ldots, n\}$. Define $\mu_{j}: S_{n} \rightarrow \mathbb{N}$ by $\mu_{j}(w)=|w|_{v_{j}}$. 
Remark 19. As a consequence of Theorem 17 (with $k=m=1, T=\mathbb{N}, M=(1), \tau=\mu_{1}$, $F=\left\{h_{\vec{x}}: \vec{x} \in \mathbb{A}^{n}\right\}$, and $p$ any idempotent in $\beta \mathbb{N}$ ) we have that whenever $D$ is piecewise syndetic in $S_{0}$ and $n \in \mathbb{N}$, there exists $w \in S_{n}$ such that $\left\{w(\vec{x}): \vec{x} \in \mathbb{A}^{n}\right\} \subseteq D$. Since whenever $S_{0}$ is finitely colored, one color class must be piecewise syndetic, we see that the multi-variable Hales-Jewett Theorem, Theorem 3, follows. And, since central sets are piecewise syndetic, we have that whenever $D$ is central in $S_{0}$ and $n \in \mathbb{N}$, there exists $w \in S_{n}$ such that $\left\{w(\vec{x}): \vec{x} \in \mathbb{A}^{n}\right\} \subseteq D$.

Theorem 20. Let $n \in \mathbb{N}$ and let $D$ be a central subset of $S_{0}$. Let $F$ be a finite nonempty set of $S_{0}$-preserving homomorphisms from $S_{n}$ into $S_{0}$. Then $\left\{w \in S_{n}:(\forall \nu \in F)(\nu(w) \in D)\right\}$ is central in $S_{n}$.

Proof. Let $T=S_{n} \cup S_{0}$ and extend each $\nu \in F$ to all of $T$ by defining $\nu$ to be the identity on $S_{0}$. By Theorem 5(2), pick a central subset $Q$ of $T$ such that for each $t \in Q$, $\{\nu(t): \nu \in F\} \subseteq D\}$. Since $S_{n}$ is an ideal of $T, Q \cap S_{n}$ is central in $S_{n}$ and $Q \cap S_{n} \subseteq$ $\left\{w \in S_{n}:(\forall \nu \in F)(\nu(w) \in D)\right\}$.

The following corollary provides sufficient conditions for applying Theorem 17.

Corollary 21. Let $m, n \in \mathbb{N}$ with $m \leqslant n$. Let $M$ be an $m \times m$ lower triangular matrix with rational entries. Assume that the entries on the diagonal are positive and the entries below the diagonal are negative or zero. Let $\vec{p}=\left(p_{1}, p_{2}, \ldots, p_{m}\right)$ be an idempotent in $\times_{i=1}^{m} \beta \mathbb{N}$. For $i \in\{1,2, \ldots, m\}$ let $\tau_{i}=\sum_{j=1}^{n} \alpha_{i, j} \mu_{j}$ where each $\alpha_{i, j} \in \mathbb{Q}$. Assume that for each $i \in\{1,2, \ldots, m\}$ we can choose $t(i) \in\{1,2, \ldots, n\}$ such that

(1) $\alpha_{i, t(i)}>0$,

(2) if $l \in\{1,2, \ldots, m\}$ and $l>i$, then $\alpha_{i, t(l)}=0$, and

(3) if $l \in\{1,2, \ldots, m\}$ and $l<i$, then $\alpha_{i, t(l)} \leqslant 0$.

Then each $\tau_{i}$ is an $S_{0}$-independent homomorphism from $S_{n}$ to $\mathbb{Q}$. Let $F$ be a nonempty finite set of $S_{0}$-preserving homomorphisms from $S_{n}$ to $S_{0}$ and let $D$ be a piecewise syndetic subset of $S_{0}$. Whenever $B_{i} \in p_{i}$ for each $i \in\{1,2, \ldots, m\}$, there exists $w \in S_{n}$ such that $\nu(w) \in D$ for each $\nu \in F$ and

$$
M\left(\begin{array}{c}
\tau_{1}(w) \\
\tau_{2}(w) \\
\vdots \\
\tau_{m}(w)
\end{array}\right) \in \times_{i=1}^{m} B_{i}
$$

Proof. Define $\psi: S_{n} \rightarrow \mathbb{Q}^{m}$ by $\psi(w)=\left(\begin{array}{c}\tau_{1}(w) \\ \vdots \\ \tau_{m}(w)\end{array}\right)$. We wish to apply Theorem 17 with $T=\mathbb{Q}$. For this we need to show that whenever $B_{i} \in p_{i}$ for $i \in\{1,2, \ldots, m\}$, there exists $\vec{z} \in \psi\left[S_{n}\right]$ such that $M \vec{z} \in \times_{i=1}^{m} B_{i}$. So let $B_{i} \in p_{i}$ for $i \in\{1,2, \ldots, m\}$. 
We show first that for each $r \in \mathbb{N}$, there exists $\vec{z} \in(r \mathbb{N})^{m}$ such that $M \vec{z} \in \times_{i=1}^{m} B_{i}$, so let $r \in \mathbb{N}$ be given. Note that $M^{-1}$ is lower triangular with positive diagonal entries and nonnegative entries below the diagonal. Probably the easiest way to see this is to solve the system of equations $M \vec{z}=\vec{x}$ by back substitution. Alternatively we may write $M=D(I+N)$ where $D$ is diagonal with positive entries and $N$ is a strictly lower triangular matrix (all of whose non-zero entries are negative) verifying $N^{m}=O$. Setting $x=-N$ in $1-x^{m}=(1-x)\left(1+x+x^{2}+\cdots x^{m-1}\right)$ gives $(I+N)^{-1}=I+\sum_{j=1}^{m-1}(-1)^{j} N^{j}$. Hence $(I+N)^{-1}$ is lower triangular with 1s along the diagonal and nonnegative entries below the diagonal. Multiplying $(I+N)^{-1}$ by $D^{-1}$ on the right gives the desired result. Let $c \in \mathbb{N}$ be such that all entries of $c M^{-1}$ are nonnegative integers. By [6, Lemma 6.6] $r c \mathbb{N} \in p_{i}$ for each $i \in\{1,2, \ldots, m\}$ so pick $x_{i} \in B_{i} \cap r c \mathbb{N}$. Letting $\vec{z}=M^{-1} \vec{x}$ one has that $\vec{z} \in(r \mathbb{N})^{m}$ and $M \vec{z} \in \times_{i=1}^{m} B_{i}$.

Now assume we have chosen $t(i)$ for $i \in\{1,2, \ldots, m\}$ as in the statement of the corollary. Pick $d \in \mathbb{N}$ such that $d \alpha_{i, j} \in \mathbb{Z}$ for each $i \in\{1,2, \ldots, m\}$ and each $j \in$ $\{1,2, \ldots, n\}$ and let $\delta_{i, j}=d \alpha_{i, j}$. Let

$$
J=\{1,2, \ldots, n\} \backslash\{t(1), t(2), \ldots, t(m)\} .
$$

Let $s=\prod_{i=1}^{m} \delta_{i, t(i)}$ and pick $r \in \mathbb{N}$ such that $s$ divides $r$ and

$$
r>\max \left\{s \sum_{j \in J}\left|\delta_{i, j}\right|: i \in\{1,2, \ldots, m\}\right\} .
$$

Pick $\vec{z} \in(r \mathbb{N})^{m}$ such that $M \vec{z} \in \times_{i=1}^{m} B_{i}$. We shall produce $w \in S_{n}$ such that $\psi(w)=\vec{z}$ by determining $\mu_{j}(w)$ for each $j \in\{1,2, \ldots, n\}$. (To be definite, we then let $w=\prod_{j=1}^{n} v_{j}^{\mu_{j}(w)}$.)

For $j \in J$, let $\mu_{j}(w)=s$. Let

$$
\mu_{t(1)}(w)=d \frac{z_{1}}{\delta_{1, t(1)}}-\sum_{j \in J} \delta_{1, j} \frac{s}{\delta_{1, t(1)}}
$$

and note that $\prod_{l=2}^{m} \delta_{l, t(l)}$ divides $\mu_{t(1)}(w)$ and by the choice of $r, \mu_{t(1)}(w)>0$, as is, of course, required. Now let $k \in\{2,3, \ldots, m\}$ and assume that for each $i \in\{1,2, \ldots, k-1\}$, we have chosen $\mu_{t(i)}(w) \in \mathbb{N}$ such that $\sum_{l=i+1}^{m} \delta_{l, t(l)}$ divides $\mu_{t(i)}(w)$. Then let

$$
\mu_{t(k)}(w)=d \frac{z_{k}}{\delta_{k, t(k)}}-\sum_{i=1}^{k-1} \frac{\delta_{k, t(i)}}{\delta_{k, t(k)}} \mu_{t(i)}(w)-\sum_{j \in J} s \frac{\delta_{k, j}}{\delta_{k, t(k)}} .
$$

Then $\mu_{t(k)}(w) \geqslant \frac{1}{\delta_{k, t(k)}}\left(d z_{k}-\sum_{j \in J} s \delta_{k, j}\right)>0$ and, if $k<m$, then $\sum_{l=k+1}^{m} \delta_{l, t(l)}$ divides $\mu_{t(k)}(w)$.

It is now a routine matter to verify that for $k \in\{1,2, \ldots, m\}$,

$$
\tau_{k}(w)=\sum_{i=1}^{k} \alpha_{k, t(i)} \mu_{t(i)}(w)+\sum_{j \in J} \alpha_{k, j} \mu_{j}(w)=z_{k} .
$$

The sufficient conditions in Corollary 21 on the coefficients $\alpha_{i, j}$ of the homomorphisms $\tau_{i}$ apply to all lower triangular matrices with positive diagonal entries and entries below the diagonal less than or equal to zero. A complete solution to the problem of which 
matrices and which $S_{0}$-independent homomorphisms satisfy the hypotheses of Theorem 17 seems quite difficult. The following simple example illustrates that one cannot get necessary and sufficient conditions on the coefficients of the homomorphisms $\tau_{i}$ valid for all lower triangular matrices with positive diagonal entries and entries below the diagonal less than or equal to zero.

Theorem 22. Let $M=\left(\begin{array}{cc}1 & 0 \\ -1 & 1\end{array}\right)$, let $N=\left(\begin{array}{ll}1 & 0 \\ 0 & 1\end{array}\right)$, let $\tau_{1}=2 \mu_{1}+\mu_{2}$ and let $\tau_{2}=\mu_{1}+2 \mu_{2}$.

(1) If $p_{1}$ and $p_{2}$ are any idempotents in $\beta \mathbb{N}, B_{1} \in p_{1}$, and $B_{2} \in p_{2}, F$ is a finite set of $S_{0}$-preserving homomorphisms from $S_{2}$ to $S_{0}$, and $D$ is a piecewise syndetic subset of $S_{0}$, then there exists $w \in S_{2}$ such that $M\left(\begin{array}{l}\tau_{1}(w) \\ \tau_{2}(w)\end{array}\right) \in B_{1} \times B_{2}$ and $\nu(w) \in D$ for each $\nu \in F$.

(2) There exist idempotents $p_{1}$ and $p_{2}$ in $\beta \mathbb{N}$ and sets $B_{1} \in p_{1}$ and $B_{2} \in p_{2}$ for which there does not exist $w \in S_{2}$ such that $N\left(\begin{array}{c}\tau_{1}(w) \\ \tau_{2}(w)\end{array}\right) \in B_{1} \times B_{2}$.

Proof. (1) Let $p_{1}$ and $p_{2}$ be idempotents in $\beta \mathbb{N}$, and let $B_{1} \in p_{1}$ and $B_{2} \in p_{2}$ be given. By Theorem 15, it suffices to show that there exists $w \in S_{2}$ such that $M\left(\begin{array}{l}\tau_{1}(w) \\ \tau_{2}(w)\end{array}\right) \in \times_{i=1}^{2} B_{i}$ By [6, Lemma 6.6], $3 \mathbb{N} \in p_{1}$ and $3 \mathbb{N} \in p_{2}$. Pick $z_{2} \in B_{2} \cap 3 \mathbb{N}$ and pick $z_{1}>z_{2}$ in $B_{1} \cap 3 \mathbb{N}$. Let $k_{1}=\frac{1}{3} z_{1}-\frac{1}{3} z_{2}$ and let $k_{2}=\frac{1}{3} z_{1}-\frac{1}{3} z_{2}$. Let $w=v_{1}^{k_{1}} v_{2}^{k_{2}}$ so that $\mu_{1}(w)=\frac{1}{3} z_{1}-\frac{1}{3} z_{2}$ and $\mu_{2}(w)=\frac{1}{3} z_{1}+\frac{2}{3} z_{2}$. Then $\tau_{1}(w)=z_{1}, \tau_{2}(w)=z_{1}+z_{2}$, and $M\left(\begin{array}{c}\tau_{1}(w) \\ \tau_{2}(w)\end{array}\right)=\left(\begin{array}{c}z_{1} \\ z_{2}\end{array}\right)$.

(2) Let $B_{1}=F S\left(\left\langle 2^{4 n}\right\rangle_{n=1}^{\infty}\right)$ and let $B_{2}=F S\left(\left\langle 2^{4 n+2}\right\rangle_{n=1}^{\infty}\right)$. By [6, Lemma 5.11] pick idempotents $p_{1}$ and $p_{2}$ in $\beta \mathbb{N}$ such that $B_{1} \in p_{1}$ and $B_{2} \in p_{2}$. Suppose we have some $w \in S_{2}$ and elements $z_{1} \in B_{1}$ and $z_{2} \in B_{2}$ such that $N\left(\begin{array}{c}\tau_{1}(w) \\ \tau_{2}(w)\end{array}\right)=\left(\begin{array}{l}z_{1} \\ z_{2}\end{array}\right)$. Then $2 z_{1}-z_{2}=3 \mu_{1}(w)>0$ and $2 z_{2}-z_{1}=3 \mu_{2}(w)>0$ so $z_{2}<2 z_{1}$ and $z_{1}<2 z_{2}$. Pick $F, G \in$ $\mathcal{P}_{f}(\mathbb{N})$ such that $z_{1}=\sum_{t \in F} 2^{4 t}$ and $z_{2}=\sum_{t \in G} 2^{4 t+2}$. Let $m=\max F$ and let $k=\max G$. Then $2^{4 m} \leqslant z_{1}<2^{4 m+1}$ and $2^{4 k+2} \leqslant z_{2}<2^{4 k+3}$. Then $2^{4 m+2}>2 z_{1}>z_{2} \geqslant 2^{4 k+2}$ so $m \geqslant k+1$. Also $2^{4 k+4}>2 z_{2}>z_{1} \geqslant 2^{4 m} \geqslant 2^{4 k+4}$, a contradiction.

Recall that a $k \times m$ matrix $M$ is image partition regular over $\mathbb{N}$ if and only if, whenever $\mathbb{N}$ is finitely colored, there is some $\vec{z} \in \mathbb{N}^{m}$ such that the entries of $M \vec{z}$ are monochromatic. This class includes all triangular (upper or lower) matrices with rational entries and positive diagonal entries. See [6, Theorem 15.24] for several characterizations of matrices that are image partition regular over $\mathbb{N}$.

Corollary 23. Let $k, m, n \in \mathbb{N}$ with $m \leqslant n$. Let $M$ be a $k \times m$ matrix with rational entries which is image partition regular over $\mathbb{N}$. Let $p$ be a minimal idempotent in $\beta \mathbb{N}$ and let $\widehat{p}=(p, p, \ldots, p) \in \times_{i=1}^{k} \beta \mathbb{N}$. Let $\sigma$ be an injection from $\{1,2, \ldots, m\}$ to $\{1,2, \ldots, n\}$. For $i \in\{1,2, \ldots, m\}$ let $\tau_{i}=\mu_{\sigma(i)}$. Let $F$ be a nonempty finite set of $S_{0}$-preserving 
homomorphisms from $S_{n}$ to $S_{0}$ and let $D$ be a piecewise syndetic subset of $S_{0}$. Then whenever $B \in p$ there exists $w \in S_{n}$ such that $\nu(w) \in D$ for each $\nu \in F$ and

$$
M\left(\begin{array}{c}
\tau_{1}(w) \\
\tau_{2}(w) \\
\vdots \\
\tau_{m}(w)
\end{array}\right) \in B^{k} .
$$

Proof. We note that the mapping

$$
w \mapsto\left(\tau_{1}(w), \tau_{2}(w), \ldots, \tau_{m}(w)\right)
$$

defines an $S_{0}$-independent homomorphism from $S_{n}$ onto $\mathbb{N}^{m}$. So in order to apply Theorem 17 , we must verify that whenever $B_{i} \in p$ for each $i \in\{1,2, \ldots, k\}$, there exists $\vec{z} \in \mathbb{N}^{m}$ such that $M \vec{z} \in \times_{i=1}^{k} B_{i}$. We then pick $w \in S_{n}$ such that $\tau_{i}(w)=z_{i}$ for each $i \in\{1,2, \ldots, m\}$, which one may do because $\sigma$ is injective.

Now $\bigcap_{i=1}^{k} B_{i} \in p$ so $B=\bigcap_{i=1}^{k} B_{i}$ is central in $\mathbb{N}$. By $[6$, Theorem $15.24(\mathrm{~h})]$ there exists $\vec{z} \in \mathbb{N}^{m}$ such that $M \vec{z} \in B^{k}$.

Corollary 23 applies to a much larger class of matrices than Corollary 21, but is more restrictive in that the same minimal idempotent must occur in each coordinate. Suppose we have a $k \times m$ matrix $M$ which is image partition regular over $\mathbb{N}$. If we knew that whenever $B_{1}, B_{2}, \ldots, B_{k}$ are central subsets of $\mathbb{N}$, there exist $\vec{z} \in \mathbb{N}^{m}$ with $M \vec{z} \in \times_{i=1}^{k} B_{i}$, then in Corollary 23 we could allow $\vec{p}=\left(p_{1}, p_{2}, \ldots, p_{k}\right)$ to be an arbitrary minimal idempotent in $\times_{i=1}^{k} \beta \mathbb{N}$. We shall see now that this fails.

Theorem 24. Let $M=\left(\begin{array}{ll}1 & 1 \\ 1 & 2\end{array}\right)$. Then $M$ is image partition regular over $\mathbb{N}$. For $x \in \mathbb{N}$ let $\phi(x)=\max \left\{t \in \omega: 2^{t} \leqslant x\right\}$ and for $i \in\{0,1,2,3\}$ let $B_{i}=\{x \in \mathbb{N}: \phi(x) \equiv i($ $\bmod 4)\}$. Then $B_{0}$ and $B_{2}$ are central and there do not exist $x$ and $y \in \mathbb{N}$ such that $M\left(\begin{array}{l}x \\ y\end{array}\right) \in B_{0} \times B_{2}$.

Proof. By [6, Theorem 15.5] $M$ is image partition regular over $\mathbb{N}$. Since $\mathbb{N}=\bigcup_{i=0}^{3} B_{i}$ some $B_{i}$ is central. But then, by [6, Lemma 15.23.2], each $B_{i}$ is central. Suppose we have some $x, y \in \mathbb{N}$ such that $M\left(\begin{array}{l}x \\ y\end{array}\right) \in B_{0} \times B_{2}$. Let $n=\phi(x+y)$. Then $2^{n} \leqslant x+y<2^{n+1}$ so $y<2^{n+1}-x$ and thus $2 y<2^{n+2}-2 x$ so $x+2 y<2^{n+2}-x<2^{n+2}$ and thus $\phi(x+2 y) \in\{n, n+1\}$.

Note also that Corollary 23 is more restrictive than Corollary 21 in that the idempotent $p$ is also required to be minimal. It is well known and easy to see that $F S\left(\left\langle 2^{2 t}\right\rangle_{t=1}^{\infty}\right)$ does not contain any three term arithmetic progression. Consequently, if

$$
M=\left(\begin{array}{ll}
1 & 0 \\
1 & 1 \\
1 & 2
\end{array}\right),
$$


then the assumption in Corollary 23 that the idempotent $p$ is minimal cannot be deleted.

Remark 25. We remarked in the introduction that we are not concerned with the instances of Theorem 4 with $m>0$ because the natural versions of our results in this section are not valid. The results in this section apply to all piecewise syndetic subsets of $S_{0}$. In particular, they apply to central subsets. It was shown in [2, Theorem 3.6] that, given $a \in \mathbb{A}$, there is a central set $D \in S_{1}$ such that there is no $w \in S_{2}$ with $\left\{w\left(a v_{1}\right), w\left(v_{1} a\right)\right\} \subseteq D$.

\section{Homomorphisms satisfying our hypotheses}

In Corollary 21 we produced $S_{0}$-independent homomorphisms from $S_{n}$ to $\mathbb{Q}$ as linear combinations of the functions $\mu_{i}$ with coefficients from $\mathbb{Q}$. We shall see in Corollary 30 that if $T$ is commutative and cancellative, then the only $S_{0}$-independent homomorphisms $\varphi: S_{n} \rightarrow T$ are of the form $\varphi(w)=\sum_{i=1}^{n} \mu_{i}(w) \cdot a_{i}$ where each $a_{i}$ is in the group of differences of $T$.

In Corollary 23 we used $S_{0}$-independent homomorphisms $\tau_{i}=\mu_{\sigma(i)}$ from $S_{n}$ to $\mathbb{N}$ and the surjection $w \mapsto\left(\tau_{1}(w), \tau_{2}(w), \ldots, \tau_{m}(w)\right)$ from $S_{n}$ onto $\mathbb{N}^{m}$. We show in Corollary 33 that if $T=\mathbb{N}$, then these are essentially the only choices for $\tau_{i}$ satisfying the hypotheses of Theorem 17.

Recall that throughout this section $\mathbb{A}$ is a fixed nonempty finite alphabet.

Definition 26. Let $n \in \mathbb{N}$. For $w \in S_{n}$, let $w^{\prime} \in\left\{v_{1}, v_{2}, \ldots, v_{n}\right\}^{+}$be obtained from $w$ by deleting all occurrences of letters belonging to $\mathbb{A}$.

Lemma 27. Fix $n \in \mathbb{N}$. Let $(T,+)$ be a cancellative semigroup and let $\varphi: S_{n} \rightarrow T$ be an $S_{0}$-independent homomorphism. Then $\varphi(w)=\varphi\left(w^{\prime}\right)$ for all $w \in S_{n}$.

Proof. It suffices to show that if $w_{1}, w_{2} \in S_{n}$ and $u \in S_{0}$, then $\varphi\left(w_{1} u w_{2}\right)=\varphi\left(w_{1} w_{2}\right)$. Let $v=v_{1} v_{2} \cdots v_{n}$. On one hand $\varphi\left(v w_{1} u w_{2} v\right)=\varphi(v)+\varphi\left(w_{1} u w_{2}\right)+\varphi(v)$, and on the other hand $\varphi\left(v w_{1} u w_{2} v\right)=\varphi\left(v w_{1} u\right)+\varphi\left(w_{2} v\right)=\varphi\left(v w_{1}\right)+\varphi\left(w_{2} v\right)=\varphi\left(v w_{1} w_{2} v\right)=\varphi(v)+$ $\varphi\left(w_{1} w_{2}\right)+\varphi(v)$. The result now follows.

For $u, w \in A^{+}$we say $u$ and $w$ are Abelian equivalent, and write $u \sim_{A b} w$, whenever $|u|_{a}=|w|_{a}$ for all $a \in A$.

Lemma 28. Fix $n \in \mathbb{N}$. Let $(T,+)$ be a cancellative and commutative semigroup and let $\varphi: S_{n} \rightarrow T$ be an $S_{0}$-independent homomorphism. For each $w_{1}, w_{2} \in S_{n}$ we have $\varphi\left(w_{1}\right)=\varphi\left(w_{2}\right)$ whenever $w_{1}^{\prime} \sim_{A b} w_{2}^{\prime}$.

Proof. Assume $w_{1}, w_{2} \in S_{n}$ and $w_{1}^{\prime} \sim_{A b} w_{2}^{\prime}$. Let $m=\left|w_{1}^{\prime}\right|=\left|w_{2}^{\prime}\right|$. We show that $\varphi\left(w_{1}^{\prime}\right)=\varphi\left(w_{2}^{\prime}\right)$ which in turn implies that $\varphi\left(w_{1}\right)=\varphi\left(w_{2}\right)$ by Lemma 27 . The result is immediate in case $n=1$ for in this case $w_{1}^{\prime}=w_{2}^{\prime}=v_{1}^{m}$. So let us assume that $n \geqslant 2$ in which case $m \geqslant 2$. Since the symmetric group on $m$-letters is generated by the 2-cycle $(1,2)$ and the $m$-cycle $(1,2, \ldots, m)$ it suffices to show

(i) If $x, w \in\left(\mathbb{A} \cup\left\{v_{1}, v_{2}, \ldots, v_{n}\right\}\right)^{+}$and $x w \in S_{n}$, then $w x \in S_{n}$ and $\varphi(w x)=\varphi(x w)$. 
(ii) Let $\varepsilon$ be the empty word. If $x, y \in\left(\mathbb{A} \cup\left\{v_{1}, v_{2}, \ldots, v_{n}\right\}\right)^{+}, w \in\left(\mathbb{A} \cup\left\{v_{1}, v_{2}, \ldots, v_{n}\right\}\right)^{+}$ $\cup\{\varepsilon\}$ and $x y w \in S_{n}$, then $y x w \in S_{n}$ and $\varphi(y x w)=\varphi(x y w)$.

Then given $l_{1}, l_{2}, \ldots, l_{m} \in \mathbb{A} \cup\left\{v_{1}, v_{2}, \ldots, v_{n}\right\}$ by (i) we have $\varphi\left(l_{1} l_{2} \cdots l_{m}\right)=$ $\varphi\left(l_{2} l_{3} \cdots l_{m} l_{1}\right)$ and by (ii) we have $\varphi\left(l_{1} l_{2} l_{3} \cdots l_{m}\right)=\varphi\left(l_{2} l_{1} l_{3} \cdots l_{m}\right)$.

To establish (i), we have $\varphi(x w)+\varphi(x w x)=\varphi(x w x w x)=\varphi(x w x)+\varphi(w x)$, whence $\varphi(x w)=\varphi(w x)$. Note that we are using here that $T$ is commutative. For (ii), let $v=$ $v_{1} v_{2} \cdots v_{n}$. Then, using (i) twice, $\varphi(v)+\varphi(x y w)+\varphi(v)=\varphi(v x y w v)=\varphi(v x)+\varphi(y w v)=$ $\varphi(x v)+\varphi(y w v)=\varphi(x v y w v)=\varphi(x v y)+\varphi(w v)=\varphi(v y x)+\varphi(w v)=\varphi(v y x w v)=$ $\varphi(v)+\varphi(y x w)+\varphi(v)$. The result now follows.

We remark that Lemma 28 does not hold in general if $T$ is not commutative. For example, consider the homomorphism $\varphi: S_{3} \rightarrow S_{2}$ where $\varphi(w)$ is the word in $S_{2}$ obtained from $w$ by deleting all occurrences of the variable $v_{3}$ in addition to all letters belonging to $\mathbb{A}$. Then $S_{2}$ is cancellative and $\varphi$ is an $S_{0}$-independent homomorphism. However, $\varphi\left(v_{1} v_{2} v_{3}\right)=v_{1} v_{2} \neq v_{2} v_{1}=\varphi\left(v_{2} v_{1} v_{3}\right)$ yet $v_{1} v_{2} v_{3} \sim_{A b} v_{2} v_{1} v_{3}$.

Theorem 29. Fix $n \in \mathbb{N}$. Let $(T,+)$ be a cancellative and commutative semigroup and let $\varphi: S_{n} \rightarrow T$ be an $S_{0}$-independent homomorphism. Then there exists a homomorphism $f: \mathbb{N}^{n} \rightarrow T$ such that $\varphi(w)=f\left(\mu_{1}(w), \mu_{2}(w), \ldots, \mu_{n}(w)\right)$ for all $w \in S_{n}$.

Proof. Define $f: \mathbb{N}^{n} \rightarrow T$ by $f\left(x_{1}, x_{2}, \ldots, x_{n}\right)=\varphi\left(v_{1}^{x_{1}} v_{2}^{x_{2}} \cdots v_{n}^{x_{n}}\right)$. By Lemma $28, f$ is as required.

Corollary 30. Let $n \in \mathbb{N}$, let $(T,+)$ be a commutative and cancellative semigroup, let $G$ be the group of differences of $T$, and let $\varphi$ be an $S_{0}$-independent homomorphism from $S_{n}$ to $T$. There exist $a_{1}, a_{2}, \ldots, a_{n}$ in $G$ such that for each $w \in S_{n}, \varphi(w)=\sum_{i=1}^{n} \mu_{i}(w) \cdot a_{i}$.

Proof. Pick a homomorphism $f: \mathbb{N}^{n} \rightarrow T$ as guaranteed by Theorem 29. For $j \in$ $\{1,2, \ldots, n\}$, define $\vec{z}^{[j]} \in \mathbb{N}^{n}$ by, for $i \in\{1,2, \ldots, n\}$,

$$
z_{i}^{[j]}= \begin{cases}2 & \text { if } i=j \\ 1 & \text { if } i \neq j\end{cases}
$$

Let $\widehat{1}=(1,1, \ldots, 1) \in \mathbb{N}^{n}$. Let $c=f(\widehat{1})$ and for $j \in\{1,2, \ldots, n\}$, let $a_{j}=f\left(\vec{z}^{[j]}\right)-c$. Then

$$
(n+1) \cdot c=f(n+1, n+1, \ldots, n+1)=\sum_{j=1}^{n} f\left(\vec{z}^{[j]}\right)=\left(\sum_{j=1}^{n} a_{j}\right)+n \cdot c
$$

so $c=\sum_{j=1}^{n} a_{j}$.

We claim that $f\left(x_{1}, x_{2}, \ldots, x_{n}\right)=\sum_{j=1}^{n} x_{j} \cdot a_{j}$ for all $\left(x_{1}, x_{2}, \ldots, x_{n}\right) \in \mathbb{N}^{n}$. To see this we proceed by induction on $\sum_{j=1}^{n} x_{j}$. If $\sum_{j=1}^{n} x_{j}=n$ then $\left(x_{1}, x_{2}, \ldots, x_{n}\right)=\widehat{1}$ whence

$$
f\left(x_{1}, x_{2}, \ldots, x_{n}\right)=c=\sum_{j=1}^{n} a_{j}=\sum_{j=1}^{n} 1 \cdot a_{j} .
$$


Next let $N \geqslant n$ and suppose that $f\left(x_{1}, x_{2}, \ldots, x_{n}\right)=\sum_{j=1}^{n} x_{j} \cdot a_{j}$ for all $\left(x_{1}, x_{2}, \ldots, x_{n}\right) \in$ $\mathbb{N}^{n}$ with $\sum_{j=1}^{n} x_{j} \leqslant N$. Let $\left(x_{1}, x_{2}, \ldots, x_{n}\right) \in \mathbb{N}^{n}$ be such that $\sum_{j=1}^{n} x_{j}=N+1$. Pick $j \in\{1,2, \ldots, n\}$ such that $x_{j} \geqslant 2$. Then

$$
f\left(x_{1}, x_{2}, \ldots, x_{n}\right)+f(\widehat{1})=f\left(x_{1}, \ldots, x_{j-1}, x_{j}-1, x_{j+1}, \ldots, x_{n}\right)+f\left(\vec{z}^{[j]}\right) .
$$

Since $f\left(\vec{z}^{[j]}\right)-f(\widehat{1})=a_{j}$ it follows by our induction hypothesis that

$$
f\left(x_{1}, x_{2}, \ldots, x_{n}\right)=\sum_{i=1}^{j-1} x_{i} \cdot a_{i}+\left(x_{j}-1\right) \cdot a_{j}+\sum_{i=j+1}^{n} x_{i} \cdot a_{i}+a_{j}=\sum_{i=1}^{n} x_{i} \cdot a_{i} .
$$

Consequently, for all $w \in S_{n}$,

$$
\varphi(w)=f\left(\mu_{1}(w), \mu_{2}(w), \ldots, \mu_{n}(w)\right)=\sum_{j=1}^{n} \mu_{j}(w) \cdot a_{j} .
$$

In the proof of the next lemma, we shall use the fact that if $n \in \mathbb{N}$, $f: \mathbb{N}^{n} \rightarrow \mathbb{N}$ is a homomorphism, $\vec{x}, \vec{y}^{[1]}, \vec{y}^{[2]}, \ldots, \vec{y}^{[n]} \in \mathbb{N}^{n}, \alpha_{1}, \alpha_{2}, \ldots, \alpha_{n} \in \mathbb{Z}$, and $\vec{x}=\sum_{i=1}^{n} \alpha_{i} \vec{y}^{[i]}$, then

$$
f(\vec{x})=\sum_{i=1}^{n} \alpha_{i} f\left(\vec{y}^{[i]}\right) .
$$

We note that if $\alpha_{i} \leqslant 0$, then $f$ is not defined at $\alpha_{i} \vec{y}^{[i]}$. So to verify the above equality, let $I=\left\{i \in\{1,2, \ldots, n\}: \alpha_{i}<0\right\}$ and let $J=\left\{i \in\{1,2, \ldots, n\}: \alpha_{i}>0\right\}$. Then

$$
\vec{x}+\sum_{i \in I}\left(-\alpha_{i}\right) \vec{y}^{[i]}=\sum_{i \in J} \alpha_{i} \vec{y}^{[i]}
$$

SO

$$
f(\vec{x})+\sum_{i \in I}\left(-\alpha_{i}\right) f\left(\vec{y}^{[i]}\right)=\sum_{i \in J} \alpha_{i} f\left(\vec{y}^{[i]}\right)
$$

So

$$
f(\vec{x})=\sum_{i \in I \cup J} \alpha_{i} f\left(\vec{y}^{[i]}\right)=\sum_{i=1}^{n} \alpha_{i} f\left(\vec{y}^{[i]}\right) .
$$

Lemma 31. Let $n \in \mathbb{N}$ and $f: \mathbb{N}^{n} \rightarrow \mathbb{N}$ be a surjective homomorphism. Then there exists $i \in\{1,2, \ldots, n\}$ such that $f(\vec{x})=x_{i}$ for each $\vec{x}=\left(x_{1}, x_{2}, \ldots, x_{n}\right) \in \mathbb{N}^{n}$, i.e., $f$ is the projection onto the $i$ 'th coordinate.

Proof. We begin by showing that $f(\widehat{1})=1$ where $\widehat{1}=(1,1, \ldots, 1)$. Since $f$ is surjective, it suffices to show that $f(\vec{x}) \geqslant f(\widehat{1})$ for each $\vec{x}=\left(x_{1}, x_{2}, \ldots, x_{n}\right) \in \mathbb{N}^{n}$. For each $r \in \mathbb{N}$ we have that

$$
r \vec{x}=(r-1) \widehat{1}+\left(1+r\left(x_{1}-1\right), 1+r\left(x_{2}-1\right), \ldots, 1+r\left(x_{n}-1\right)\right) .
$$

It follows that $r f(\vec{x})=f(r \vec{x})>f((r-1) \widehat{1})=(r-1) f(\widehat{1})$ or equivalently that $r(f(\vec{x})-$ $f(\widehat{1}))>-f(\widehat{1})$. As $r$ is arbitrary we deduce that $f(\vec{x})-f(\widehat{1}) \geqslant 0$ as claimed.

For each $j \in\{1,2, \ldots, n\}$ let $\vec{z}^{[j]}=\left(z_{1}^{[j]}, z_{2}^{[j]}, \ldots, z_{n}^{[j]}\right) \in \mathbb{N}^{n}$ be as in (1). As $\sum_{j=1}^{n} \vec{z}^{[j]}=$ $(n+1) \widehat{1}$ we have $\sum_{j=1}^{n} f\left(\vec{z}^{[j]}\right)=f((n+1) \widehat{1})=n+1$. It follows that there exists a unique $k \in\{1,2, \ldots, n\}$ such that $f\left(\vec{z}^{[k]}\right)=2$ and $f\left(\vec{z}^{[j]}\right)=1$ for all $j \neq k$. Without loss of generality, we may assume that $f\left(\vec{z}^{[1]}\right)=2$ and $f\left(\vec{z}^{[j]}\right)=1$ for all $j \in\{2,3, \ldots n\}$. 
Let $\vec{x}=\left(x_{1}, x_{2}, \ldots, x_{n}\right) \in \mathbb{N}^{n}$. We will show that $f(\vec{x})=x_{1}$. We first note that

$$
(n+1) \vec{x}=\sum_{i=1}^{n}\left(n x_{i}-\sum_{\substack{j=1 \\ j \neq i}}^{n} x_{j}\right) \vec{z}^{[i]} .
$$

Therefore

$$
(n+1) f(\vec{x})=\left(n x_{1}-\sum_{j=2}^{n} x_{j}\right) \cdot 2+\sum_{i=2}^{n}\left(n x_{i}-\sum_{\substack{j=1 \\ j \neq i}}^{n} x_{j}\right) \cdot 1
$$

and thus $(n+1) f(\vec{x})=(n+1) x_{1}$.

Corollary 32. Let $n, m \in \mathbb{N}$. For $i \in\{1,2, \ldots, n\}$ and $j \in\{1,2, \ldots, m\}$ let $\pi_{i}: \mathbb{N}^{n} \rightarrow \mathbb{N}$ and $\pi_{j}^{\prime}: \mathbb{N}^{m} \rightarrow \mathbb{N}$ denote the projections onto the $i$ 'th and $j^{\prime}$ th coordinates respectively. Assume that $f: \mathbb{N}^{n} \rightarrow \mathbb{N}^{m}$ is a surjective homomorphism. For $i \in\{1,2, \ldots, m\}$, let $f_{i}=\pi_{i}^{\prime} \circ f$. Then there is an injection $\sigma:\{1,2, \ldots, m\} \rightarrow\{1,2, \ldots, n\}$ such that for each $i \in\{1,2, \ldots, m\}, f_{i}=\pi_{\sigma(i)}$. In particular $m \leqslant n$.

Proof. By hypothesis each $f_{i}: \mathbb{N}^{n} \rightarrow \mathbb{N}$ is a surjective homomorphism. Therefore by Lemma 31, there exists a mapping $\sigma:\{1,2, \ldots, m\} \rightarrow\{1,2, \ldots, n\}$ such that $f_{i}(\vec{x})=$ $\pi_{\sigma(i)}(\vec{x})=x_{\sigma(i)}$ for each $\vec{x} \in \mathbb{N}^{n}$. But as $f$ is surjective, it follows that $\sigma$ is injective.

Corollary 33. Let $n, m \in \mathbb{N}$. For each $i \in\{1,2, \ldots, m\}$ let $\tau_{i}: S_{n} \rightarrow \mathbb{N}$ be an $S_{0^{-}}$ independent homomorphism. If the mapping $w \mapsto\left(\tau_{1}(w), \tau_{2}(w), \ldots, \tau_{m}(w)\right)$ takes $S_{n}$ onto $\mathbb{N}^{m}$, then there exists an injection $\sigma:\{1,2, \ldots, m\} \rightarrow\{1,2, \ldots, n\}$ such that $\tau_{i}=\mu_{\sigma(i)}$ for each $i \in\{1,2, \ldots, m\}$. In particular we must have $m \leqslant n$.

Proof. By Theorem 29, for each $i \in\{1,2, \ldots, m\}$, pick a homomorphism $f_{i}: \mathbb{N}^{n} \rightarrow \mathbb{N}$ such that $\tau_{i}(w)=f_{i}\left(\mu_{1}(w), \mu_{2}(w), \ldots, \mu_{n}(w)\right)$ for each $w \in S_{n}$. Define $f: \mathbb{N}^{n} \rightarrow \mathbb{N}^{m}$ by $f(\vec{x})=\left(f_{1}(\vec{x}), f_{2}(\vec{x}), \ldots, f_{m}(\vec{x})\right)$. We claim that $f$ is surjective, so let $\vec{y} \in \mathbb{N}^{m}$ be given and pick $w \in S_{n}$ such that $\left(\tau_{1}(w), \tau_{2}(w), \ldots, \tau_{m}(w)\right)=\vec{y}$. For $j \in\{1,2, \ldots, n\}$, let $x_{j}=|w|_{v_{j}}$. Then $f(\vec{x})=\vec{y}$.

By Corollary 32, pick an injection $\sigma:\{1,2, \ldots, m\} \rightarrow\{1,2, \ldots, n\}$ such that for each $i \in\{1,2, \ldots, m\}, f_{i}=\pi_{\sigma(i)}$. Let $w \in S_{n}$ be given and let $\vec{x}=\left(|w|_{v_{1}},|w|_{v_{2}}, \ldots,|w|_{v_{n}}\right)$, Then for $i \in\{1,2, \ldots, m\}, \tau_{i}(w)=f_{i}(\vec{x})=x_{\sigma(i)}=|w|_{v_{\sigma(i)}}$.

\section{Compact subsemigroups of $(\beta \mathbb{N})^{k}$}

Besides the idempotents in $\phi\left[\beta S_{n}\right]$, there are more ultrafilters that satisfy Corollary 12 , and they form a compact semigroup.

Theorem 34. Let $n \in \mathbb{N}$, let $C$ be a compact right topological semigroup, let $\phi: S_{n} \rightarrow C$ be an $S_{0}$-independent homomorphism for which $\phi\left[S_{n}\right]$ is contained in the topological center of $C$, denote also by $\phi$ the continuous extension from $\beta S$ to $C$, and let $F$ be a finite nonempty set of $S_{0}$-preserving homomorphisms from $S_{n}$ into $S_{0}$. Let

$$
\begin{aligned}
P=\left\{p \in \phi\left[\beta S_{n}\right]:\right. & \text { for every neighborhood } U \text { of } p \\
& \text { and every piecewise syndetic subset } D \text { of } S_{0} \\
& \left.\left(\exists w \in S_{n}\right)(\phi(w) \in U \text { and }(\forall \nu \in F)(\nu(w) \in D))\right\} .
\end{aligned}
$$

Then $P$ is a compact subsemigroup of $C$ containing all the idempotents of $\phi\left[\beta S_{n}\right]$. 
Proof. It is clear that $P$ is compact. By Corollary 12, $P$ contains all the idempotents in $\phi\left[\beta S_{n}\right]$. To see that $P$ is a subsemigroup of $C$, let $p, q \in P$. Let $U$ be an open neighborhood of $p q$ and let $D$ be a piecewise syndetic subset of $S_{0}$. By [6, Theorem 4.43] pick $s \in S_{0}$ such that $s^{-1} D$ is central and pick a minimal idempotent $r$ in $\beta S_{0}$ such that $s^{-1} D \in r$. Pick a neighborhood $V$ of $p$ such that $\rho_{q}[V] \subseteq U$. Since $\left(s^{-1} D\right)^{\star} \in r$, it is piecewise syndetic so pick $w \in S_{n}$ such that $\phi(w) \in V$ and $\nu(w) \in\left(s^{-1} D\right)^{\star}$ for each $\nu \in F$.

Then $\phi(w) q \in U$ and $\phi(w)$ is in the topological center of $C$ so pick a neighborhood $Q$ of $q$ such that $\lambda_{\phi(w)}[Q] \subseteq U$. For each $\nu \in F, \nu(w)^{-1}\left(s^{-1} D\right)^{\star} \in r$. Let $E=\bigcap_{\nu \in F} \nu(w)^{-1}\left(s^{-1} D\right)^{\star}$. Then $E \in r$ so $E$ is piecewise syndetic in $S_{0}$. Pick $u \in S_{n}$ such that $\phi(u) \in Q$ and $\nu(u) \in E$ for each $\nu \in F$. Then $\phi(s w u)=\phi(w) \phi(u) \in U$ and for each $\nu \in F, \nu(s w u)=s \nu(w) \nu(u) \in D$.

In the next results we focus on the semigroup of natural numbers $\mathbb{N}$ and $S_{0}$-independent homomorphisms from $S_{n}$ onto $\mathbb{N}^{m}$, so by Corollary 33 we may assume that we have $m \leqslant n$ and are dealing with $S_{0}$-independent homomorphisms $\tau_{i}$ from $S_{n}$ to $\mathbb{N}$ defined by $\tau_{i}(w)=|w|_{v_{\sigma(i)}}$ for some injection $\sigma:\{1,2, \ldots, m\} \rightarrow\{1,2, \ldots, n\}$.

We consider the collection of all $k$-tuples of ultrafilters that satisfy Theorem 17 , in the case where $T=\mathbb{N}$ and where the homomorphisms are of the special form $h_{\vec{x}}$.

Definition 35. Let $k, m, n \in \mathbb{N}$ with $m \leqslant n$, let $M$ be a $k \times m$ matrix with entries from $\mathbb{Q}$, let $F$ be a finite nonempty set of $S_{0}$-preserving homomorphisms from $S_{n}$ to $S_{0}$, and let $\sigma$ be an injection from $\{1,2, \ldots, m\}$ to $\{1,2, \ldots, n\}$.

$$
\begin{aligned}
P_{M, F, \sigma}=\left\{\vec{p} \in \times_{i=1}^{k} \beta \mathbb{N}:\right. & \text { whenever } D \text { is a piecewise syndetic subset of } S_{0} \\
& \text { and for all } i \in\{1,2, \ldots, k\}, B_{i} \in p_{i}, \text { there exists } \\
& w \in S_{n} \text { such that }(\forall \nu \in F)(\nu(w) \in D) \text { and } \\
& \left.M\left(\begin{array}{c}
\mu_{\sigma(1)}(w) \\
\vdots \\
\mu_{\sigma(m)}(w)
\end{array}\right) \in \times_{i=1}^{k} B_{i}\right\}
\end{aligned}
$$

Recall that for $\vec{x} \in \mathbb{A}^{n}$ we have defined the $S_{0}$-preserving homomorphism $h_{\vec{x}}: S_{n} \rightarrow S_{0}$ by $h_{\vec{x}}(w)=w(\vec{x})$. We are particularly interested in the set $\left\{h_{\vec{x}}: \vec{x} \in \mathbb{A}^{n}\right\}$ because of the relationship with the Hales-Jewett Theorem. We see now that if $F=\left\{h_{\vec{x}}: \vec{x} \in \mathbb{A}^{n}\right\}$, then $P_{M, F, \sigma}$ does not depend on $\sigma$. We keep $\sigma$ in the notation because there are $S_{0}$-preserving homomorphisms which are not of the form $h_{\vec{x}}$.

Lemma 36. Let $k, m, n \in \mathbb{N}$ with $m \leqslant n$, let $M$ be a $k \times m$ matrix with entries from $\mathbb{Q}$, let $F=\left\{h_{\vec{x}}: \vec{x} \in \mathbb{A}^{n}\right\}$, and let $\sigma$ and $\eta$ be injections from $\{1,2, \ldots, m\}$ to $\{1,2, \ldots, n\}$. Then $P_{M, F, \sigma}=P_{M, F, \eta}$.

Proof. It suffices to show that $P_{M, F, \sigma} \subseteq P_{M, F, \eta}$, so let $\vec{p} \in P_{M, F, \sigma}$. To see that $\vec{p} \in P_{M, F, \eta}$, let $D$ be a piecewise syndetic subset of $S_{0}$ and for $i \in\{1,2, \ldots, k\}$, let $B_{i} \in p_{i}$. Pick $w \in S_{n}$ such that for all $\vec{x} \in \mathbb{A}^{n}, h_{\vec{x}}(w) \in D$ and $M\left(\begin{array}{c}\mu_{\sigma(1)}(w) \\ \vdots \\ \mu_{\sigma(m)}(w)\end{array}\right) \in \times_{i=1}^{k} B_{i}$. 
Define $\delta:\{\sigma(1), \sigma(2), \ldots, \sigma(m)\} \rightarrow\{1,2, \ldots, n\}$ by letting $\delta(\sigma(i))=\eta(i)$ for $i \in$ $\{1,2, \ldots, m\}$ and extend $\delta$ to a permutation of $\{1,2, \ldots, n\}$. Define $w^{\prime} \in S_{n}$ by $w^{\prime}=$ $w\left(v_{\delta(1)} v_{\delta(2)} \cdots v_{\delta(n)}\right)$. Then for $j \in\{1,2, \ldots, n\}, \mu_{j}(w)=\mu_{\delta(j)}\left(w^{\prime}\right)$ so for $i \in\{1,2, \ldots, m\}$, $\mu_{\sigma(i)}(w)=\mu_{\delta(\sigma(i))}\left(w^{\prime}\right)=\mu_{\eta(i)}\left(w^{\prime}\right)$ and thus

$$
M\left(\begin{array}{c}
\mu_{\eta(1)}\left(w^{\prime}\right) \\
\vdots \\
\mu_{\eta(m)}\left(w^{\prime}\right)
\end{array}\right)=M\left(\begin{array}{c}
\mu_{\sigma(1)}(w) \\
\vdots \\
\mu_{\sigma(m)}(w)
\end{array}\right) \in \times_{i=1}^{k} B_{i} .
$$

Now let $\vec{x} \in \mathbb{A}^{n}$ be given and define $\vec{z} \in \mathbb{A}^{n}$ by letting $z_{i}=x_{\delta(i)}$ for $i \in\{1,2, \ldots, n\}$. Then $h_{\vec{x}}\left(w^{\prime}\right)=h_{\vec{z}}(w) \in D$.

If one lets $C=(\beta \mathbb{N})^{k}$ and defines $\phi$ on $S_{n}$ by $\phi(w)=M\left(\begin{array}{c}\mu_{\sigma(1)}(w) \\ \vdots \\ \mu_{\sigma(m)}(w)\end{array}\right)$, one may not be able to invoke Theorem 34 to conclude that $P_{M, F, \sigma}$ is a semigroup because $\phi$ may not take $S_{n}$ to $C$. Consider, for example, $M=\left(\begin{array}{c}1 \\ -1\end{array}\right)$.

Theorem 37. Let $k, m, n \in \mathbb{N}$ with $m \leqslant n$, let $M$ be a $k \times m$ matrix with entries from $\mathbb{Q}$, and let $F$ be a finite nonempty set of $S_{0}$-preserving homomorphisms from $S_{n}$ to $S_{0}$. Let $\sigma$ be an injection from $\{1,2, \ldots, m\}$ to $\{1,2, \ldots, n\}$. If $P_{M, F, \sigma} \neq \varnothing$, then $P_{M, F, \sigma}$ is a compact subsemigroup of $(\beta \mathbb{N})^{k}$.

Proof. Assume that $P_{M, F, \sigma} \neq \varnothing$. We begin by showing that $P_{M, F, \sigma}$ is compact. Let $\vec{p}=\left(p_{1}, p_{2}, \ldots, p_{k}\right) \in(\beta \mathbb{N})^{k} \backslash P_{M, F, \sigma}$ and pick piecewise syndetic $D \subseteq S_{0}$ and $B_{i} \in p_{i}$ for each $i \in\{1,2, \ldots, k\}$ such that there is no $w \in S_{n}$ with $\nu(w) \in D$ for all $\nu \in F$ and $M\left(\begin{array}{c}\mu_{\sigma(1)}(w) \\ \vdots \\ \mu_{\sigma(m)}(w)\end{array}\right) \in \times_{i=1}^{k} B_{i}$; then $\times_{i=1}^{k} \overline{B_{i}}$ is a neighborhood of $\vec{p}$ which misses $P_{M, F, \sigma}$ so $P_{M, F, \sigma}$ is closed and hence compact.

To see that $P_{M, F, \sigma}$ is a semigroup, let $\vec{p}, \vec{q} \in P_{M, F, \sigma}$. Let $D$ be a piecewise syndetic subset of $S_{0}$ and for each $i \in\{1,2, \ldots, k\}$, let $B_{i} \in p_{i}+q_{i}$. By [6, Theorem 4.43], pick $s \in S_{0}$ such that $s^{-1} D$ is central in $S_{0}$ and pick a minimal idempotent $r \in \beta S_{0}$ such that $s^{-1} D \in r$. For each $i \in\{1,2, \ldots, k\}$, let $C_{i}=\left\{x \in \mathbb{N}:-x+B_{i} \in q_{i}\right\}$ and note that $C_{i} \in p_{i}$. Then as $\left(s^{-1} D\right)^{\star} \in r$, we deduce that $\left(s^{-1} D\right)^{\star}$ is central and hence in particular piecewise syndetic. Since $\vec{p} \in P_{M, F, \sigma}$, pick $w \in S_{n}$ such that $\nu(w) \in\left(s^{-1} D\right)^{\star}$ for all $\nu \in F$ and $M\left(\begin{array}{c}\mu_{\sigma(1)}(w) \\ \vdots \\ \mu_{\sigma(m)}(w)\end{array}\right)=\vec{z} \in \times_{i=1}^{k} C_{i}$. Let $G=\bigcap_{\nu \in F} \nu(w)^{-1}\left(s^{-1} D\right)^{\star}$. Then $G \in r$ so $G$ is piecewise syndetic in $S_{0}$. Also $\vec{q} \in P_{M, F, \sigma}$ and for each $i \in\{1,2, \ldots, k\}$, 
$-z_{i}+B_{i} \in q_{i}$ so pick $u \in S_{n}$ such that $\nu(u) \in G$ for each $\nu \in F$ and $M\left(\begin{array}{c}\mu_{\sigma(1)}(u) \\ \vdots \\ \mu_{\sigma(m)}(u)\end{array}\right)=$ $\vec{y} \in \times_{i=1}^{k}\left(-z_{i}+B_{i}\right)$.

Given $\nu \in F, \nu(w u)=\nu(w) \nu(u) \in s^{-1} D$ so $\nu(s w u)=s \nu(w u) \in D$. Finally $M\left(\begin{array}{c}\mu_{\sigma(1)}(s w u) \\ \vdots \\ \mu_{\sigma(m)}(s w u)\end{array}\right)=M\left(\begin{array}{c}\mu_{\sigma(1)}(w u) \\ \vdots \\ \mu_{\sigma(m)}(w u)\end{array}\right)=M\left(\begin{array}{c}\mu_{\sigma(1)}(w)+\mu_{\sigma(1)}(u) \\ \vdots \\ \mu_{\sigma(m)}(w)+\mu_{\sigma(m)}(u)\end{array}\right)=\vec{z}+\vec{y} \in$ $\times_{i=1}^{k} B_{i}$.

Corollary 38. Let $m, n \in \mathbb{N}$ with $m \leqslant n$. Let $M$ be an $m \times m$ lower triangular matrix with rational entries. Assume that the entries on the diagonal are positive and the entries below the diagonal are negative or zero. Let $F$ be a finite nonempty set of $S_{0}$-preserving homomorphisms from $S_{n}$ to $S_{0}$. Let $\sigma$ be an injection from $\{1,2, \ldots, m\}$ to $\{1,2, \ldots, n\}$. Then $P_{M, F, \sigma}$ is a compact subsemigroup of $(\beta \mathbb{N})^{m}$ containing the idempotents of $(\beta \mathbb{N})^{m}$.

Proof. Let $k=m$. By Corollary 21, $P_{M, F, \sigma}$ contains the idempotents of $(\beta \mathbb{N})^{k}$ so in particular $P_{M, F, \sigma} \neq \varnothing$. The result now follows by Theorem 37 .

Corollary 39. Let $k, m, n \in \mathbb{N}$ with $m \leqslant n$. Let $M$ be $a k \times m$ matrix with rational entries which is image partition regular over $\mathbb{N}$. Let $F$ be a finite nonempty set of $S_{0}$-preserving homomorphisms from $S_{n}$ to $S_{0}$. Let $\sigma$ be an injection from $\{1,2, \ldots, m\}$ to $\{1,2, \ldots, n\}$. Then $P_{M, F, \sigma}$ is a compact subsemigroup of $(\beta \mathbb{N})^{k}$ containing $\left\{(p, p, \ldots, p) \in(\beta \mathbb{N})^{k}: p\right.$ is a minimal idempotent of $\beta \mathbb{N}\}$.

Proof. By Corollary 23, $P_{M, F, \sigma}$ contains $\left\{(p, p, \ldots, p) \in(\beta \mathbb{N})^{k}: p\right.$ is a minimal idempotent of $\beta \mathbb{N}\}$ so Theorem 37 applies.

If $M=\left(\begin{array}{ll}1 & 1 \\ 1 & 2\end{array}\right)$ and $F$ is a finite nonempty set of $S_{0}$-preserving homomorphisms from $S_{n}$ to $S_{0}$, then by Corollary 39 we have that $P_{M, F, \sigma}$ contains $\{(p, p): p$ is a minimal idempotent of $\beta \mathbb{N}\}$ but by Theorem $24, P_{M, F, \sigma}$ does not contain $\left\{\left(p_{1}, p_{2}\right): p_{1}\right.$ and $p_{2}$ are minimal idempotents of $\beta \mathbb{N}\}$.

Given a finite coloring of a semigroup, at least one of the color classes must be piecewise syndetic, so results concluding that piecewise syndetic sets have a certain property guarantee the corresponding conclusion for finite colorings. We see now a situation where the conclusions are equivalent - a fact that has interesting consequences for piecewise syndetic sets and for colorings.

The following result is analgous to a well know property of piecewise syndetic sets of natural numbers. That is, a translation invariant family $\mathcal{S}$ of finite nonempty subsets of $\mathbb{N}$ is partition regular if and only if every piecewise syndetic subset of $\mathbb{N}$ contains a member of $\mathcal{S}$.

Theorem 40. Let $n \in \mathbb{N}$, let $\tau$ be an $S_{0}$-independent homomorphism from $S_{n}$ to $\mathbb{N}$, and let $B \subseteq \mathbb{N}$. The following statements are equivalent. 
(a) Whenever $S_{0}$ is finitely colored, there exists $w \in S_{n}$ such that $\left\{w(\vec{x}): x \in \mathbb{A}^{n}\right\}$ is monochromatic and $\tau(w) \in B$.

(b) Whenever $D$ is a piecewise syndetic subset of $S_{0}$, there exists $w \in S_{n}$ such that $\left\{w(\vec{x}): \vec{x} \in \mathbb{A}^{n}\right\} \subseteq D$ and $\tau(w) \in B$.

Proof. It is trivial that (b) implies (a), so assume that (a) holds and let $D$ be a piecewise syndetic subset of $S_{0}$. Note that for each $r \in \mathbb{N}$, there is some $m \in \mathbb{N}$ such that whenever the length $m$ words in $S_{0}$ are $r$-colored, there is some $w \in S_{n}$ of length $m$ such that $\left\{w(\vec{x}): \vec{x} \in \mathbb{A}^{n}\right\}$ is monochromatic and $\tau(w) \in B$. (If there is a bad $r$-coloring $\varphi_{m}$ of the length $m$ words for each $m$ then $\bigcup_{m=1}^{\infty} \varphi_{m}$ is a bad $r$-coloring of $S_{0}$.)

Since $D$ is piecewise syndetic, pick finite nonempty $G \subseteq S_{0}$ such that for every finite nonempty subset $H$ of $S_{0}$ there exists $s \in S_{0}$ with $H s \subseteq \bigcup_{t \in G} t^{-1} D$. Let $r=|G|$ and pick $m \in \mathbb{N}$ such that whenever the length $m$ words in $S_{0}$ are $r$-colored, there is some $w \in S_{n}$ such that $\left\{w(\vec{x}): \vec{x} \in \mathbb{A}^{n}\right\}$ is monochromatic and $\tau(w) \in B$. Let $H$ be the set of length $m$ words in $S_{0}$ and pick $s \in S_{0}$ such that $H s \subseteq \bigcup_{t \in G} t^{-1} D$. For $u \in H$ pick $\varphi(u) \in G$ such that $u s \in \varphi(u)^{-1} G$. Pick $w \in S_{n}$ of length $m$ and $t \in G$ such that for all $\vec{x} \in \mathbb{A}^{n}$, $\varphi(w(\vec{x}))=t$ and $\tau(w) \in B$. Let $w^{\prime}=t w s$. Then for $\vec{x} \in \mathbb{A}^{n}, w^{\prime}(\vec{x})=t(w(\vec{x})) s \in D$ and $\tau\left(w^{\prime}\right)=\tau(w) \in B$.

If $n=1$, the following corollary yields the statement in the second paragraph of the abstract.

Corollary 41. Let $n \in \mathbb{N}$, let $\tau$ be an $S_{0}$-independent homomorphism from $S_{n}$ onto $\mathbb{N}$, and let $Q=\left\{p \in \beta \mathbb{N}\right.$ : whenever $S_{0}$ is finitely colored and $B \in p$, there exists $w \in S_{n}$ such that $\left\{w(\vec{x}): \vec{x} \in \mathbb{A}^{n}\right\}$ is monochromatic and $\left.\tau(w) \in B\right\}$. Then $Q$ is a compact subsemigroup of $\beta \mathbb{N}$ containing all of the idempotents.

Proof. Let $k=m=1$, let $M=(1)$, and let $F=\left\{h_{\vec{x}}: \vec{x} \in \mathbb{A}^{n}\right\}$. By Corollary 33 , pick $\sigma(1) \in\{1,2, \ldots, n\}$ such that $\tau=\mu_{\sigma(1)}$. By Corollary 38, $P_{M, F, \sigma}$ is a compact subsemigroup of $\beta \mathbb{N}$ containing all of the idempotents and by Theorem $40, Q=P_{M, F, \sigma}$.

Recall that a set of sets $\mathcal{B}$ is said to be partition regular if whenever $\mathcal{F}$ is a finite set of sets and $\bigcup \mathcal{F} \in \mathcal{B}$, there exist $A \in \mathcal{F}$ and $B \in \mathcal{B}$ such that $B \subseteq A$.

Corollary 42. Let $n \in \mathbb{N}$ and let $\tau$ be an $S_{0}$-independent homomorphism from $S_{n}$ to $\mathbb{N}$. Let $\mathcal{B}=\left\{B \subseteq \mathbb{N}\right.$ : whenever $D$ is a piecewise syndetic subset of $S_{0}$, there exists $w \in S_{n}$ such that $\left\{w(\vec{x}): \vec{x} \in \mathbb{A}^{n}\right\} \subseteq D$ and $\left.\tau(w) \in B\right\}$. Then $\mathcal{B}$ is partition regular.

Proof. By Theorem $40, \mathcal{B}=\left\{B \subseteq \mathbb{N}\right.$ : whenever $S_{0}$ is finitely colored, there exists $w \in S_{n}$ such that $\left\{w(\vec{x}): x \in \mathbb{A}^{n}\right\}$ is monochromatic and $\left.\tau(w) \in B\right\}$. It is routine to show that if $k \in \mathbb{N}, B_{i} \subseteq \mathbb{N}$ for each $i \in\{1,2, \ldots, k\}$, and $\bigcup_{i=1}^{k} B_{i}$ has the property that whenever $S_{0}$ is finitely colored, there exists $w \in S_{n}$ such that $\left\{w(\vec{x}): x \in \mathbb{A}^{n}\right\}$ is monochromatic and $\tau(w) \in \bigcup_{i=1}^{k} B_{i}$, then some $B_{i} \in \mathcal{B}$.

Since the intersection of any collection of compact semigroups having the finite intersection property is a compact semigroup, it follows that there exists a smallest compact subsemigroup of $(\beta \mathbb{N})^{k}$ containing the idempotents of $(\beta \mathbb{N})^{k}$. 
Question 43. Let $k \in \mathbb{N}$, let $M$ be the $k \times k$ identity matrix, and let $\sigma$ be the identity function on $\{1,2, \ldots, k\}$.

(a) If $F=\left\{h_{\vec{x}}: \vec{x} \in \mathbb{A}^{k}\right\}$, is $P_{M, F, \sigma}$ the smallest compact subsemigroup of $(\beta \mathbb{N})^{k}$ containing the idempotents of $(\beta \mathbb{N})^{k}$ ?

(b) If not, does there exist a finite nonempty set $F$ of $S_{0}$-preserving homomorphisms such that $P_{M, F, \sigma}$ is the smallest compact subsemigroup of $(\beta \mathbb{N})^{k}$ containing the idempotents of $(\beta \mathbb{N})^{k}$ ?

Question 44. Let $k \in \mathbb{N}$ and let $M$ and $N$ be $k \times k$ lower triangular matrices with rational entries, positive diagonal entries, and nonpositive entries below the diagonal. Do there exist a finite nonempty set $F$ of $S_{0}$-preserving homomorphisms from $S_{k}$ to $S_{0}$ and a permutation $\sigma$ of $\{1,2, \ldots, k\}$ such that $P_{M, F, \sigma} \neq P_{N, F, \sigma}$ ?

Because of Question 43, we are interested in the smallest compact subsemigroup of $(\beta \mathbb{N})^{k}$ containing the idempotents of $(\beta \mathbb{N})^{k}$.

Given a compact right topological semigroup $T$, recall that we let $E(T)$ be the set of idempotents in $T$ and $K(T)$ is the smallest ideal of $T$. If $I$ is a set and for each $i \in I$, $T_{i}$ is a compact right topological semigroup, then $E\left(\times_{i \in I} T_{i}\right)=\times_{i \in I} E\left(T_{i}\right)$ because the operation in $\times_{i \in I} T_{i}$ is coordinatewise. Also by $\left[6\right.$, Theorem 2.23] $K\left(\times_{i \in I} T_{i}\right)=\times_{i \in I} K\left(T_{i}\right)$ so that $E\left(K\left(\times_{i \in I} T_{i}\right)\right)=\times_{i \in I} E\left(K\left(T_{i}\right)\right)$.

Definition 45. Let $T$ be a compact right topological semigroup and let $A \subseteq T$. Then $J_{T}(A)$ is the smallest compact subsemigroup of $T$ containing $A$.

We next show that $J_{(\beta \mathbb{N})^{k}}\left(E\left((\beta \mathbb{N})^{k}\right)\right)=\left(J_{\beta \mathbb{N}}(E(\beta \mathbb{N}))\right)^{k}$ for $k \in \mathbb{N}$ and that a similar result applies to the minimal idempotents. Notice that in general $J_{T_{1} \times T_{2}}\left(A_{1} \times A_{2}\right) \subseteq$ $J_{T_{1}}\left(A_{1}\right) \times J_{T_{2}}\left(A_{2}\right)$. But equality need not always hold even in the case that $T_{1}=T_{2}$ and $A_{1}=A_{2}$. For example, let $A^{+}$be the free semigroup on the alphabet $A=\{a, b\}$, and $T=\beta A^{+}$. Then, identifying the letters of $A$ with the length one words so that $A$ is a subset of $T$, we have $J_{T}(A) \times J_{T}(A)=T \times T$ while $J_{T \times T}(A \times A)=c \ell_{T \times T}\{(u, w) \in$ $\left.A^{+} \times A^{+}:|u|=|w|\right\}$.

Theorem 46. Let $T_{1}$ and $T_{2}$ be compact right topological semigroups and for $i \in\{1,2\}$ let $A_{i}$ be a nonempty subset of $T_{i}$ with $A_{i} \subseteq\left\{a b: a, b \in A_{i}\right\}$. Then $J_{T_{1} \times T_{2}}\left(A_{1} \times A_{2}\right)=$ $J_{T_{1}}\left(A_{1}\right) \times J_{T_{2}}\left(A_{2}\right)$.

Proof. As $J_{T_{1}}\left(A_{1}\right) \times J_{T_{2}}\left(A_{2}\right)$ is a compact subsemigroup of $T_{1} \times T_{2}$ containing $A_{1} \times A_{2}$ we have immediately that $J_{T_{1} \times T_{2}}\left(A_{1} \times A_{2}\right) \subseteq J_{T_{1}}\left(A_{1}\right) \times J_{T_{2}}\left(A_{2}\right)$. So it remains to show that $J_{T_{1}}\left(A_{1}\right) \times J_{T_{2}}\left(A_{2}\right) \subseteq J_{T_{1} \times T_{2}}\left(A_{1} \times A_{2}\right)$. Let $Y=\left\{q \in J_{T_{2}}\left(A_{2}\right):(p, q) \in J_{T_{1} \times T_{2}}\left(A_{1} \times\right.\right.$ $\left.A_{2}\right)$ for all $\left.p \in A_{1}\right\}$. Then $Y$ is compact and $A_{2} \subseteq Y$. Further, let $q_{1}, q_{2} \in Y$ and $p \in A_{1}$, and write $p=p_{1} p_{2}$ with $p_{1}, p_{2} \in A_{1}$. Then $\left(p_{1}, q_{1}\right),\left(p_{2}, q_{2}\right) \in J_{T_{1} \times T_{2}}\left(A_{1} \times A_{2}\right)$ and hence $\left(p_{1}, q_{1}\right)\left(p_{2}, q_{2}\right)=\left(p, q_{1} q_{2}\right) \in J_{T_{1} \times T_{2}}\left(A_{1} \times A_{2}\right)$. Thus $Y$ is a compact subsemigroup of $J_{T_{2}}\left(A_{2}\right)$ containing $A_{2}$ so $Y=J_{T_{2}}\left(A_{2}\right)$. 
Now let $X=\left\{x \in J_{T_{1}}\left(A_{1}\right):\{x\} \times J_{T_{2}}\left(A_{2}\right) \subseteq J_{T_{1} \times T_{2}}\left(A_{1} \times A_{2}\right)\right\}$. Then $X$ is compact and if $p \in A_{1}$, then $\{p\} \times J_{T_{2}}\left(A_{2}\right)=\{p\} \times Y \subseteq J_{T_{1} \times T_{2}}\left(A_{1} \times A_{2}\right)$, so $A_{1} \subseteq X$. We next claim that $X$ is a semigroup. In fact, let $x_{1}, x_{2} \in X$ and set $Z=\left\{z \in J_{T_{2}}\left(A_{2}\right):\left(x_{1} x_{2}, z\right) \in\right.$ $\left.J_{T_{1} \times T_{2}}\left(A_{1} \times A_{2}\right)\right\}$. Then $Z$ is compact. Let $q \in A_{2}$ and write $q=q_{1} q_{2}$ with $q_{1}, q_{2} \in A_{2}$. Then $\left(x_{1}, q_{1}\right),\left(x_{2}, q_{2}\right) \in J_{T_{1} \times T_{2}}\left(A_{1} \times A_{2}\right)$ and hence $\left(x_{1} x_{2}, q\right) \in J_{T_{1} \times T_{2}}\left(A_{1} \times A_{2}\right)$. Thus $Z$ contains $A_{2}$. Finally, let $z_{1}, z_{2} \in Z$. Then since $z_{1}, z_{2} \in J_{T_{2}}\left(A_{2}\right)$ and $x_{1}, x_{2} \in X$ we deduce that $\left(x_{1}, z_{1}\right),\left(x_{2}, z_{2}\right) \in J_{T_{1} \times T_{2}}\left(A_{1} \times A_{2}\right)$ implying that $\left(x_{1} x_{2}, z_{1} z_{2}\right) \in J_{T_{1} \times T_{2}}\left(A_{1} \times A_{2}\right)$ and hence $z_{1} z_{2} \in Z$. Thus $Z$ is a compact subsemigroup of $J_{T_{2}}\left(A_{2}\right)$ containing $A_{2}$ and hence $Z=J_{T_{2}}\left(A_{2}\right)$ from which it follows that $x_{1} x_{2} \in X$. Having shown that $X$ is compact subsemigroup of $J_{T_{1}}\left(A_{1}\right)$ containing $A_{1}$ we deduce that $X=J_{T_{1}}\left(A_{1}\right)$. In conclusion, $J_{T_{1}}\left(A_{1}\right) \times J_{T_{2}}\left(A_{2}\right)=X \times J_{T_{2}}\left(A_{2}\right) \subseteq J_{T_{1} \times T_{2}}\left(A_{1} \times A_{2}\right)$ as required.

Notice in particular that if for $i \in\{1,2\}, A_{i}$ is a nonempty subset of $E\left(T_{i}\right)$, then $A_{i} \subseteq\left\{a b: a, b \in A_{i}\right\}$, so $J_{T_{1} \times T_{2}}\left(A_{1} \times A_{2}\right)=J_{T_{1}}\left(A_{1}\right) \times J_{T_{2}}\left(A_{2}\right)$.

Corollary 47. Let $k \in \mathbb{N}$. The smallest compact subsemigroup of $(\beta \mathbb{N})^{k}$ containing the idempotents of $(\beta \mathbb{N})^{k}$ is $\left(J_{\beta \mathbb{N}}(E(\beta \mathbb{N}))\right)^{k}$. The smallest compact subsemigroup of $(\beta \mathbb{N})^{k}$ containing the minimal idempotents of $(\beta \mathbb{N})^{k}$ is $\left(J_{\beta \mathbb{N}}(E(K(\beta \mathbb{N})))\right)^{k}$.

Proof. By Theorem 46 and induction,

$$
J_{(\beta \mathbb{N})^{k}}\left((E(\beta \mathbb{N}))^{k}\right)=\left(J_{\beta \mathbb{N}}(E(K(\beta \mathbb{N})))\right)^{k}
$$

and we already observed that the set of idempotents of $(\beta \mathbb{N})^{k}$ is $(E(\beta \mathbb{N}))^{k}$. The second conclusion is proved in the same way.

We note now that the version of Theorem 46 for infinite products is also valid.

Theorem 48. Let $I$ be an infinite set. For each $i \in I$, let $T_{i}$ be a compact right topological semigroup and let $A_{i}$ be a nonempty subset of $T_{i}$ such that $A_{i} \subseteq\left\{a b: a, b \in A_{i}\right\}$. Then $J_{\times_{i \in I} T_{i}}\left(\times_{i \in I} A_{i}\right)=\times_{i \in I} J_{T_{i}}\left(A_{i}\right)$.

Proof. Let $Y=\times_{i \in I} T_{i}$. For each $i \in I$, choose $e_{i} \in A_{i}$. Given $F \in \mathcal{P}_{f}(I)$, let $Y_{F}=$ $\times_{i \in F} T_{i}$, let $Z_{F}=\times_{i \in I \backslash F} T_{i}$, let

$$
X_{F}=\left\{\vec{x} \in \times_{i \in I} J_{T_{i}}\left(A_{i}\right):(\forall i \in I \backslash F)\left(x_{i}=e_{i}\right)\right\},
$$

and let $B_{F}=\left\{\vec{x} \in \times_{i \in I} A_{i}:(\forall i \in I \backslash F)\left(x_{i}=e_{i}\right)\right\}$.

We shall show that for each $F \in \mathcal{P}_{f}(I), X_{F} \subseteq J_{Y}\left(\times_{i \in I} A_{i}\right)$. Let $F \in \mathcal{P}_{f}(I)$ be given. Now $X_{F}$ is topologically and algebraically isomorphic to

$$
\times_{i \in F} J_{T_{i}}\left(A_{i}\right) \times \times_{i \in I \backslash F}\left\{e_{i}\right\},
$$

$B_{F}$ is topologically and algebraically isomorphic to $\times_{i \in F} A_{i} \times \times_{i \in I \backslash F}\left\{e_{i}\right\}$, and $\times_{i \in I \backslash F}\left\{e_{i}\right\}$ $\subseteq J_{Z_{F}}\left(\times_{i \in I \backslash F}\left\{e_{i}\right\}\right)$. So using Theorem 46 we have

$$
\begin{aligned}
X_{F} & \approx \times_{i \in F} J_{T_{i}}\left(A_{i}\right) \times \times_{i \in I \backslash F}\left\{e_{i}\right\} \\
& \subseteq J_{Y_{F}}\left(\times_{i \in F} A_{i}\right) \times J_{Z_{F}}\left(\times_{i \in I \backslash F}\left\{e_{i}\right\}\right) \\
& =J_{Y_{F} \times Z_{F}}\left(\times_{i \in F} A_{i} \times \times_{i \in I \backslash F}\left\{e_{i}\right\}\right) \\
& \approx J_{Y}\left(B_{F}\right) \\
& \subseteq J_{Y}\left(\times_{i \in I} A_{i}\right) .
\end{aligned}
$$


Next we claim that $\times_{i \in I} J_{T_{i}}\left(A_{i}\right) \subseteq c \ell_{Y} \bigcup_{F \in \mathcal{P}_{f}(I)} X_{F}$. To see this, let $\vec{z} \in \times_{i \in I} J_{T_{i}}\left(A_{i}\right)$ and let $U$ be a neighborhood of $\vec{z}$ in $Y$. Pick $F \in \mathcal{P}_{f}(I)$ and for each $i \in F$, pick a neighborhood $V_{i}$ of $z_{i}$ in $T_{i}$ such that $\bigcap_{i \in F} \pi_{i}^{-1}\left[V_{i}\right] \subseteq U$. Define $\vec{x} \in Y$ by $x_{i}=$ $\left\{\begin{array}{ll}z_{i} & \text { if } i \in F \\ e_{i} & \text { if } i \in I \backslash F .\end{array}\right.$ Then $\vec{x} \in U \cap X_{F}$. Therefore $\times_{i \in I} J_{T_{i}}\left(A_{i}\right) \subseteq J_{Y}\left(\times_{i \in I} A_{i}\right)$. Since $\times_{i \in I} J_{T_{i}}\left(A_{i}\right)$ is a compact semigroup containing $\times_{i \in I} A_{i}$, the reverse inclusion is immediate.

The curious reader may wonder what the situation is with respect to the smallest semigroup containing a given set. Given a semigroup $T$ and a nonempty subset $A$ of $T$, let $J_{T}^{\prime}(A)$ be the smallest subsemigroup of $T$ containing $A$, that is the set of all finite products of members of $A$ in any order allowing repetition. If $T_{1}$ and $T_{2}$ are any semigroups and $A_{1}$ and $A_{2}$ are nonempty subsets of $T_{1}$ and $T_{2}$ respectively such that $A_{i} \subseteq\left\{a b: a, b \in A_{i}\right\}$ for $i \in\{1,2\}$, then $J_{T_{1} \times T_{2}}^{\prime}\left(A_{1} \times A_{2}\right)=J_{T_{1}}^{\prime}\left(A_{1}\right) \times J_{T_{2}}^{\prime}\left(A_{2}\right)$. This follows from the proof of Theorem 46 by omitting all references to the topology.

However, the analogue of Theorem 48 need not hold. To see this, let $T$ be the set of words over the alphabet $\left\{a_{n}: n \in \mathbb{N}\right\}$ that have no adjacent occurrences of the same letter. Given $u, w \in T$, then let $u \cdot w$ be ordinary concatenation unless $u=x a_{n}$ and $w=a_{n} y$ for some $n \in \mathbb{N}$ and some $x, y \in T \cup\{\varnothing\}$, in which case $u \cdot w=x a_{n} y$. Let $A$ be the set of idempotents in $T$, that is $A$ is the set of length one words. Then $J_{T}^{\prime}(A)=T$ but $\left\{\vec{x} \in \times_{n=1}^{\infty} T:\left\{\left|x_{n}\right|: n \in \mathbb{N}\right\}\right.$ is bounded $\}$ is a proper subsemigroup of $\times_{n=1}^{\infty} T$ containing the idempotents.

\section{Compact ideals of $(\beta S)^{k}$}

In this section we deal with results related to the Hales-Jewett Theorem and its extensions. The first result here is motivated by the following known result that characterizes image partition regular matrices.

Theorem 49. Let $k, m \in \mathbb{N}$ and let $M$ be a $k \times m$ matrix with entries from $\mathbb{Q}$. The following statements are equivalent.

(a) $M$ is image partition regular over $\mathbb{N}$.

(b) For every central subset $D$ of $\mathbb{N}$, there exists $\vec{x} \in \mathbb{N}^{m}$ such that $M \vec{x} \in D^{k}$.

(c) For every central subset $D$ of $\mathbb{N},\left\{\vec{x} \in \mathbb{N}^{m}: M \vec{x} \in D^{k}\right\}$ is central in $\mathbb{N}^{m}$.

Proof. These are statements (a), (h), and (i) of [6, Theorem 15.24].

We now investigate ideals related to the extensions of the Hales-Jewett Theorem.

Definition 50. For $n \in \mathbb{N}$,

$$
R_{n}=\left\{p \in \beta S_{0}:(\forall B \in p)\left(\exists w \in S_{n}\right)\left(\left\{w(\vec{x}): \vec{x} \in \mathbb{A}^{n}\right\} \subseteq B\right)\right\} .
$$


There are numerous ways to use known results to show that each $R_{n} \neq \varnothing$. From the point of view of this paper, probably the easiest way is to invoke Theorem 17 as discussed above.

Theorem 51. Let $n \in \mathbb{N}$. Then $R_{n}$ is a compact two sided ideal of $\beta S_{0}$.

Proof. We have that $R_{n} \neq \varnothing$ and it is trivially compact. Let $p \in R_{n}$ and let $q \in \beta S_{0}$. To see that $R_{n}$ is a left ideal, let $B \in q p$. Pick $u \in S_{0}$ such that $u^{-1} B \in p$ and pick $w \in S_{n}$ such that $\left\{w(\vec{x}): \vec{x} \in \mathbb{A}^{n}\right\} \subseteq u^{-1} B$. Then $u w \in S_{n}$ and $\left\{(u w)(\vec{x}): \vec{x} \in \mathbb{A}^{n}\right\} \subseteq B$.

To see that $R_{n}$ is a right ideal, let $B \in p q$. Pick $w \in S_{n}$ such that $\left\{w(\vec{x}): \vec{x} \in \mathbb{A}^{n}\right\} \subseteq$ $\left\{u \in S_{0}: u^{-1} B \in q\right\}$. Pick $u \in \bigcap_{\vec{x} \in \mathbb{A}^{n}} w(\vec{x})^{-1} B$. Then $w u \in S_{n}$ and $\left\{(w u)(\vec{x}): \vec{x} \in \mathbb{A}^{n}\right\} \subseteq$ $B$.

Theorem 52. Let $n \in \mathbb{N}$. Then $R_{n+1} \subseteq R_{n}$.

Proof. Let $p \in R_{n+1}$ and let $B \in p$. Pick $w \in S_{n+1}$ such that $\left\{w(\vec{x}): \vec{x} \in \mathbb{A}^{n+1}\right\} \subseteq B$. Define $u \in S_{n}$ by $u=w\left(v_{1}, v_{2}, \ldots, v_{n}, v_{n}\right)$. Then given $\vec{x} \in \mathbb{A}^{n}, u(\vec{x})=w\left(x_{1}, x_{2}, \ldots, x_{n}, x_{n}\right) \in$ $B$.

Lemma 53. For each $r, n \in \mathbb{N}$ there exists $m \in \mathbb{N}$ such that for all $k \geqslant m$, if $S\left(\begin{array}{l}k \\ 0\end{array}\right)$ is $r$-colored, then there exists $w \in S\left(\begin{array}{c}k \\ n\end{array}\right)$ such that $\left\{w(\vec{x}): \vec{x} \in \mathbb{A}^{n}\right\}$ is monochromatic.

Proof. Let $r, n \in \mathbb{N}$. By Theorem 3, whenever $S_{0}$ is $r$-colored, there exists $w \in S_{n}$ such that $\left\{w(\vec{x}): \vec{x} \in \mathbb{A}^{n}\right\}$ is monochromatic. As in the proof of Theorem 40, pick $m \in \mathbb{N}$ such that whenever $S\left(\begin{array}{c}m \\ 0\end{array}\right)$ is $r$-colored, there exists $w \in S\left(\begin{array}{c}m \\ n\end{array}\right)$ such that $\left\{w(\vec{x}): \vec{x} \in \mathbb{A}^{n}\right\}$ is monochromatic. Let $k>m$ and pick $c \in \mathbb{A}$. Let $\varphi: S\left(\begin{array}{l}k \\ 0\end{array}\right) \rightarrow\{1,2, \ldots, r\}$ and define $\psi: S\left(\begin{array}{c}m \\ 0\end{array}\right) \rightarrow\{1,2, \ldots, r\}$ by $\psi(u)=\varphi\left(u c^{k-m}\right)$. Pick $w \in S\left(\begin{array}{c}m \\ n\end{array}\right)$ such that $\psi$ is constant on $\left\{w(\vec{x}): \vec{x} \in \mathbb{A}^{n}\right\}$. Define $u \in S\left(\begin{array}{l}k \\ n\end{array}\right)$ by $u=w c^{k-m}$. Then $\varphi$ is constant on $\left\{u(\vec{x}): \vec{x} \in \mathbb{A}^{n}\right\}$.

Theorem 54. $c \ell K\left(\beta S_{0}\right) \subsetneq \bigcap_{n=1}^{\infty} R_{n}$.

Proof. That $c \ell K\left(\beta S_{0}\right) \subseteq \bigcap_{n=1}^{\infty} R_{n}$ is an immediate consequence of Theorem 51 .

Let $B=\bigcup_{k=1}^{\infty} S\left(\begin{array}{c}k ! \\ 0\end{array}\right)$. We claim first that $B$ is not piecewise syndetic, so that $\bar{B} \cap$ $c \ell K\left(\beta S_{0}\right)=\varnothing$. We need to show that there is no $G \in \mathcal{P}_{f}\left(S_{0}\right)$ such that for all $F \in \mathcal{P}_{f}\left(S_{0}\right)$ there exists $x \in S_{0}$ such that $F x \subseteq \bigcup_{t \in G} t^{-1} B$. Suppose we have such $G$ and let $m=$ $\max \{|t|: t \in G\}$, let $r=m$ !, pick $b \in \mathbb{A}$, and let $F=\left\{b^{r}, b^{2 r}\right\}$. Pick $t, s \in G$ and $x \in S_{0}$ such that $t b^{r} x \in B$ and $s b^{2 r} x \in B$. Then $\left|t b^{r} x\right|=n$ ! for some $n>m$ and $\left|s b^{2 r} x\right|=k$ ! for some $k$. Now $k !=\left|s b^{2 r} x\right|=\left|s b^{r}\right|+n !-|t|>n !$ so $k ! \geqslant(n+1)$ ! so $\left|s b^{r}\right|+n !-|t| \geqslant(n+1)$ !. Thus $n \cdot n !=(n+1) !-n !<\left|s b^{r}\right|=|s|+r \leqslant m+m !<n+n !$, a contradiction. 
Now let $n \in \mathbb{N}$. We will show that $\bar{B} \cap R_{n} \neq \varnothing$. Let $\mathcal{R}=\left\{D \subseteq S_{0}\right.$ : whenever $D$ is finitely colored, there exists $w \in S_{n}$ such that $\left\{w(\vec{x}): \vec{x} \in \mathbb{A}^{n}\right\}$ is monochromatic $\}$. Notice that $\mathcal{R}$ is partition regular. It suffices to show that $B \in \mathcal{R}$, for then by [6, Theorem 3.11] there exists $p \in \beta S_{0}$ such that $B \in p$ and $p \subseteq \mathcal{R}$ so that $p \in \bar{B} \cap R_{n}$. So let $r \in \mathbb{N}$ and let $\varphi: B \rightarrow\{1,2, \ldots, r\}$. Pick $m$ as guaranteed by Lemma 53 for $r$ and $n$. The $\varphi$ is an $r$-coloring of $S\left(\begin{array}{c}m ! \\ 0\end{array}\right)$ so pick $w \in S\left(\begin{array}{c}m ! \\ n\end{array}\right)$ such that $\left\{w(\vec{x}): \vec{x} \in \mathbb{A}^{n}\right\}$ is monochromatic.

Since $\left\{\bar{B} \cap R_{n}: n \in \mathbb{N}\right\}$ is a collection of closed sets with the finite intersection property, we have that $\bar{B} \cap \bigcap_{n=1}^{\infty} R_{n} \neq \varnothing$.

We will need the following result.

Theorem 55 (Deuber, Prömel, Rothschild, and Voigt). Let $n, r \in \mathbb{N}$. There exist $m \in \mathbb{N}$ and $C_{n, r} \subseteq S\left(\begin{array}{c}m \\ 0\end{array}\right)$ such that

(1) there does not exist $w \in S\left(\begin{array}{c}m \\ n+1\end{array}\right)$ with $\left\{w(\vec{x}): \vec{x} \in \mathbb{A}^{n+1}\right\} \subseteq C_{n, r}$ and

(2) whenever $C_{n, r}$ is r-colored, there exists $w \in S\left(\begin{array}{c}m \\ n\end{array}\right)$ such that $\left\{w(\vec{x}): \vec{x} \in \mathbb{A}^{n}\right\}$ is monochromatic.

Proof. This is the "main theorem" of [3].

Theorem 56. Let $n \in \mathbb{N}$. Then $R_{n+1} \subsetneq R_{n}$.

Proof. For each $r \in \mathbb{N}$ pick $m(r)$ and $C_{n, r}$ as guaranteed for $r$ and $n$ by Theorem 55 . Choose an increasing sequence $\left\langle r_{i}\right\rangle_{i=1}^{\infty}$ such that the sequence $\left\langle m\left(r_{i}\right)\right\rangle_{i=1}^{\infty}$ is strictly increasing and let $D_{i}=C_{n, r_{i}}$ for each $i$. Let $E=\bigcup_{i=1}^{\infty} D_{i}$. There does not exist $w \in S_{n+1}$ such that $\left\{w(\vec{x}): \vec{x} \in \mathbb{A}^{n+1}\right\} \subseteq E$ because any such $w$ would have to have length $m\left(r_{i}\right)$ for some $i$, and then one would have $\left\{w(\vec{x}): \vec{x} \in \mathbb{A}^{n+1}\right\} \subseteq C_{n, r_{i}}$. Thus $\bar{E} \cap R_{n+1}=\varnothing$.

As in the proof of Theorem 54, let $\mathcal{R}=\left\{D \subseteq S_{0}\right.$ : whenever $D$ is finitely colored, there exists $w \in S_{n}$ such that $\left\{w(\vec{x}): \vec{x} \in \mathbb{A}^{n}\right\}$ is monochromatic $\}$. It suffices to show that $E \in \mathcal{R}$ so let $k \in \mathbb{N}$ and let $\varphi: E \rightarrow\{1,2, \ldots, k\}$. Pick $i$ such that $r_{i} \geqslant k$. Then $\varphi_{\mid D_{i}}: D_{i} \rightarrow\left\{1,2, \ldots, r_{i}\right\}$ so pick $w \in S\left(\begin{array}{c}m\left(r_{i}\right) \\ n\end{array}\right)$ such that $\varphi$ is constant on $\{w(\vec{x}): \vec{x} \in$ $\left.\mathbb{A}^{n}\right\}$.

\section{A simpler proof of an infinitary extension}

We set out in this section to provide a proof of [2, Theorem 2.12] applied to the simpler description of $n$-variable words which we have been using. As defined in this paper, what is called the set of $n$-variable words in [2], is what we call the set of strong $n$-variable words where we take $D=E=\{e\}$ in [2], take the function $T_{e}$ to be the identity, and 
let $v_{n}=\left(e, \nu_{n}\right)$ for $n \in \mathbb{N}$. As we remarked earlier, in [2, Theorem 5.1] it was shown that the version of the Graham-Rothschild that we are using here is sufficient to derive the full original version as used in [2] and [4]. Using that simplified notion, Corollary 69 implies [2, Theorem 2.12] and has a vastly simpler proof. Because of the different, and more complicated, notation used in [2], we don't feel that it is appropriate to state [2, Theorem 2.12] here. It reads essentially like Corollary 69 below.

The first few results apply to an arbitrary nonempty alphabet $\mathbb{A}$. For the results of this section, except for Corollary 70, we do not need to assume that $\mathbb{A}$ is finite.

Definition 57. For $n \in \mathbb{N}, T_{n}$ is the free semigroup over $\mathbb{A} \cup\left\{v_{1}, v_{2}, \ldots, v_{n}\right\}$. Also we set $T_{0}=S_{0}$ and $T=\bigcup_{i \in \omega} T_{i}$.

Clearly $T$ is a semigroup. Note that for $n \in \mathbb{N}$, the set $S_{n}$ of $n$-variable words is a proper subset of $T_{n}$ and that $T_{n} \subseteq T_{n+1}$.

For $u=l_{1} l_{2} \cdots l_{m} \in T$ with $|u|=m$ we define $h_{u}: T \rightarrow T$ by stating that $h_{u}(w)$ is the result of replacing each occurrence of $v_{i}$ in $w$ by $l_{i}$ for $i \in\{1,2, \ldots, m\}$. (Thus, if $w \in S_{m}$, $h_{u}(w)=w(u)$ as defined in Definition 2.) Denote also by $h_{u}$ the continuous extension of $h_{u}$ taking $\beta T$ to $\beta T$. Observe that, if $u \in \widetilde{S}\left(\begin{array}{c}m \\ k\end{array}\right)$, then $h_{u}\left[T_{m}\right] \subseteq T_{k}$.

Definition 58. For $\alpha \in \mathbb{N} \cup\{\omega\}$, a reductive sequence of height $\alpha$ over $\mathbb{A}$ is a sequence of minimal idempotents $\left\langle p_{t}\right\rangle_{t<\alpha}$ with $p_{t} \in E\left(K\left(\beta S_{t}\right)\right)$ such that for each $i, j \in \omega$ with $0 \leqslant j<i<\alpha$ one has $p_{i} \leqslant p_{j}$ and $h_{u}\left(p_{i}\right)=p_{j}$ for each $u \in \widetilde{S}\left(\begin{array}{l}i \\ j\end{array}\right)$.

It is a consequence of Corollary 68 below that reductive sequences of arbitrary height exist.

Lemma 59. Let $i \in \omega$. Then $K\left(\beta S_{i}\right)=K\left(\beta T_{i}\right)$.

Proof. We have that $S_{i}$ is an ideal of $T_{i}$ so by [6, Corollary 4.18] $\beta S_{i}$ is an ideal of $\beta T_{i}$. Therefore $K\left(\beta T_{i}\right) \subseteq \beta S_{i}$ so that by [6, Theorem 1.65] $K\left(\beta S_{i}\right)=K\left(\beta T_{i}\right)$.

Lemma 60. Let $k, m \in \omega$ with $k<m$ and let $p \in E\left(\beta S_{k}\right)$. There exists $q \in E\left(K\left(\beta S_{m}\right)\right)$ such that $q<p$.

Proof. We have $\beta S_{m} \cup \beta S_{k} \subseteq \beta T_{m}$. Pick $q \in E\left(K\left(\beta T_{m}\right)\right)$ such that $q \leqslant p$. By Lemma $59, q \in K\left(\beta S_{m}\right)$ and since $\beta S_{k} \cap \beta S_{m}=\varnothing, q \neq p$.

Lemma 61. Let $\alpha \in \mathbb{N} \cup\{\omega\}$ and let $\left\langle p_{t}\right\rangle_{t<\alpha}$ be a reductive sequence of height $\alpha$. For each $t<\alpha, p_{t} \in E\left(K\left(\beta \widetilde{S}_{t}\right)\right)$.

Proof. Since each $p_{t}$ is an idempotent, it suffices to show that $p_{t} \in K\left(\beta \widetilde{S}_{t}\right)$. Given $t<\alpha$, $\widetilde{S}_{t}$ is a right ideal of $S_{t}$ so $\beta \widetilde{S}_{t}$ is a right ideal of $\beta S_{t}$ and thus $K\left(\beta \widetilde{S}_{t}\right) \cap K\left(\beta S_{t}\right) \neq \varnothing$ so that by $\left[6\right.$, Theorem 1.65], $K\left(\beta \widetilde{S}_{t}\right)=\beta \widetilde{S}_{t} \cap K\left(\beta S_{t}\right)$. Thus it suffices to show that each $p_{t} \in \beta \widetilde{S}_{t}$. We proceed by induction on $t$. For $t=0$, we have $p_{0} \in \beta S_{0}=\beta \widetilde{S}_{0}$. Now assume that 
$t+1<\alpha$ and $p_{t} \in \beta \widetilde{S}_{t}$. We need to show that $\widetilde{S}_{t+1} \in p_{t+1}$. We begin by observing that if $w \in \widetilde{S}_{t}$ then $S_{t+1} \subseteq w^{-1} \widetilde{S}_{t+1}$ from which it follows that $\widetilde{S}_{t} \subseteq\left\{w \in T_{t+1}: w^{-1} \widetilde{S}_{t+1} \in p_{t+1}\right\}$. Now since $\widetilde{S}_{t} \in p_{t}$ we have that $\left\{w \in T_{t+1}: w^{-1} \widetilde{S}_{t+1} \in p_{t+1}\right\} \in p_{t}$ or equivalently that $\widetilde{S}_{t+1} \in p_{t} p_{t+1}$. The result now follows from the fact that $p_{t+1} \leqslant p_{t}$ and hence in particular $p_{t+1}=p_{t} p_{t+1}$.

We now introduce some new notation. We fix a nonempty (possibly infinite) alphabet $\mathbb{A}$ together with an infinite sequence of symbols $\left\{x_{1}, x_{2}, x_{3}, \ldots\right\}$ each of which is not a member of $\mathbb{A} \cup\left\{v_{i}: i \in \mathbb{N}\right\}$. We let $\mathbb{A}^{(0)}=\mathbb{A}$ and for $m \in \mathbb{N}$, we let $\mathbb{A}^{(m)}=\mathbb{A} \cup$ $\left\{x_{1}, x_{2}, \ldots, x_{m}\right\}$. For each $m \in \omega$ we let $S^{(m)}$ denote the free semigroup over $\mathbb{A}^{(m)}$. For each $i \in \mathbb{N}$ we let $S_{i}^{(m)}$ denote the set of all $i$-variable words over the alphabet $\mathbb{A}^{(m)}$ and let $\widetilde{S}_{i}^{(m)}$ denote the set of all strong $i$-variable words over $\mathbb{A}^{(m)} \cdot T_{i}^{(j)}$ will denote the free semigroup of all words over the alphabet $\mathbb{A}^{(j)} \cup\left\{v_{1}, v_{2}, \ldots, v_{i}\right\}$. Also, for each $j \in \omega$, let $S_{0}^{(j)}=\widetilde{S}_{0}^{(j)}=T_{0}^{(j)}=S^{(j)}$, and let $T^{(j)}=\bigcup_{i=1}^{\infty} T_{i}^{(j)}$. Then $T^{(j)}$ is the set of all words over $\mathbb{A}^{(j)} \cup\left\{v_{i}: i \in \mathbb{N}\right\}$.

To each $u=u_{1} u_{2} \cdots u_{m} \in T^{(j)}$ with $|u|=m$ we associate a morphism $h_{u}: \bigcup_{j \in \omega} T^{(j)} \rightarrow$ $\bigcup_{j \in \omega} T^{(j)}$ where for each $w \in \bigcup_{j \in \omega} T^{(j)}, h_{u}(w)$ is obtained from $w$ by replacing each occurrence of $v_{i}$ in $w$ by $u_{i}$ for each $i \in\{1,2, \ldots, m\}$. We also denote by $h_{u}$ its continuous extension taking $\beta\left(\bigcup_{j \in \omega} T^{(j)}\right)$ to $\beta\left(\bigcup_{j \in \omega} T^{(j)}\right)$. Also, for each $i, j \in \mathbb{N}$ we define the morphism, $\tau_{i}^{(j)}: T^{(j)} \rightarrow T^{(j-1)}$ where $\tau_{i}^{(j)}(w)$ is the word obtained from $w$ by replacing every occurrence of $x_{j}$ by $v_{i}$ and leaving all other symbols unchanged. We also denote by $\tau_{i}^{(j)}$ the continuous extension of $\tau_{i}^{(j)}$ taking $\beta T^{(j)}$ to $\beta T^{(j-1)}$. Note that $\tau_{i}^{(j)}\left[T_{i-1}^{(j)}\right]=$ $T_{i}^{(j-1)}$ and the restriction of $\tau_{i}^{(j)}$ to $T_{i-1}^{(j)}$ is an isomorphism onto $T_{i}^{(j-1)}$. Consequently the restriction of $\tau_{i}^{(j)}$ to $\beta T_{i-1}^{(j)}$ is an isomorphism onto $\beta T_{i}^{(j-1)}$.

Lemma 62. Let $m \in \omega$, let $i \in \mathbb{N} \backslash\{1\}$, and assume that $p_{i-1}^{(m)} \in \beta S_{i-1}^{(m)}$ and $p_{i-1}^{(m+1)} \in$ $K\left(\beta S_{i-1}^{(m+1)}\right)$. Then

$$
G_{i}^{(m)}=p_{i-1}^{(m)} \tau_{i}^{(m+1)}\left(p_{i-1}^{(m+1)}\right) \beta S_{i}^{(m)} \cap \beta S_{i}^{(m)} \tau_{i}^{(m+1)}\left(p_{i-1}^{(m+1)}\right) p_{i-1}^{(m)}
$$

is a group contained in $K\left(\beta S_{i}^{(m)}\right)$.

Proof. We will show that $G_{i}^{(m)}$ is the intersection of a minimal right ideal and a minimal left ideal of $\beta S_{i}^{(m)}$ and hence by [6, Theorem 1.61], $G_{i}^{(m)}$ is a group contained in $K\left(\beta S_{i}^{(m)}\right)$. Notice first that $\beta S_{i-1}^{(m)} \cup \beta S_{i}^{(m)} \subseteq \beta T_{i}^{(m)}$ so that the products $p_{i-1}^{(m)} \tau_{i}^{(m+1)}\left(p_{i-1}^{(m+1)}\right)$ and $\tau_{i}^{(m+1)}\left(p_{i-1}^{(m+1)}\right) p_{i-1}^{(m)}$ are computed in $\beta T_{i}^{(m)}$.

Since $\tau_{i}^{(m+1)}$ is an isomorphism on $\beta T_{i-1}^{(m+1)}$, we have $\tau_{i}^{(m+1)}\left[K\left(\beta T_{i-1}^{(m+1)}\right)\right]$ $=K\left(\beta T_{i}^{(m)}\right)$. Since $p_{i-1}^{(m+1)} \in K\left(\beta S_{i-1}^{(m+1)}\right)$ and $K\left(\beta S_{i-1}^{(m+1)}\right)=K\left(\beta T_{i-1}^{(m+1)}\right)$ (by Lemma 59 with the underlying alphabet taken to be $\left.\mathbb{A}^{(m+1)}\right)$, it follows that $\tau_{i}^{(m+1)}\left(p_{i-1}^{(m+1)}\right) \in$ $K\left(\beta T_{i}^{(m)}\right)$. Since $p_{i-1}^{(m)} \in \beta T_{i-1}^{(m)} \subseteq \beta T_{i}^{(m)}$, we have that $p_{i-1}^{(m)} \tau_{i}^{(m+1)}\left(p_{i-1}^{(m+1)}\right) \in K\left(\beta T_{i}^{(m)}\right)=$ $K\left(\beta S_{i}^{(m)}\right)$ so that $p_{i-1}^{(m)} \tau_{i}^{(m+1)}\left(p_{i-1}^{(m+1)}\right) \beta S_{i}^{(m)}$ is a minimal right ideal of $\beta S_{i}^{(m)}$. Similarly $\beta S_{i}^{(m)} \tau_{i}^{(m+1)}\left(p_{i-1}^{(m+1)}\right) p_{i-1}^{(m)}$ is a minimal left ideal of $\beta S_{i}^{(m)}$. 
Definition 63. For $\alpha \in \mathbb{N} \cup\{\omega\}$, a reductive array of height $\alpha$ over $\mathbb{A}$ is an $\alpha \times \omega$ array of minimal idempotents $\left\langle p_{i}^{(m)}\right\rangle_{i<\alpha}^{m<\omega}$ with $p_{i}^{(m)} \in E\left(K\left(\beta S_{i}^{(m)}\right)\right)$ satisfying the following conditions:

1. For each $m \in \omega$ the sequence $\left\langle p_{i}^{(m)}\right\rangle_{i<\alpha}$ is a reductive sequence of height $\alpha$ over $\mathbb{A}^{(m)}$.

2. $\tau_{1}^{(m)}\left(p_{0}^{(m)}\right)=p_{1}^{(m-1)}$ for each $m \in \mathbb{N}$.

3. For each $m \in \omega$ and $i<\alpha$ with $i \geqslant 2, p_{i}^{(m)}$ is the identity of the group

$$
G_{i}^{(m)}=p_{i-1}^{(m)} \tau_{i}^{(m+1)}\left(p_{i-1}^{(m+1)}\right) \beta S_{i}^{(m)} \cap \beta S_{i}^{(m)} \tau_{i}^{(m+1)}\left(p_{i-1}^{(m+1)}\right) p_{i-1}^{(m)}
$$

Definition 64. Let $i, j, m \in \omega$, with $j<i . X_{i, j}^{(m)}$ will denote the set of words in $\widetilde{S}^{(m)}\left(\begin{array}{l}i \\ j\end{array}\right)$ in which $v_{j}$ occurs only as the last letter.

Lemma 65. Let $m, n \in \mathbb{N}$ and let $\vec{p}=\left\langle p_{0}, p_{1}, p_{2}, \ldots, p_{n}\right\rangle$ be a reductive sequence of height $n+1$ over $\mathbb{A}^{(m)}$. Let $p_{n+1}$ be a minimal idempotent in $\beta S_{n+1}^{(m)}$ for which $p_{n+1} \leqslant p_{n}$. Let $j \in \omega$ with $j \leqslant n$ and let $u=u_{1} u_{2} \cdots u_{n+1} \in \widetilde{S}^{(m)}\left(\begin{array}{c}n+1 \\ j\end{array}\right)$.

(1) If $u \notin X_{n+1, j}^{(m)}$, then $h_{u}\left(p_{n+1}\right)=p_{j}$.

(2) If $u \in X_{n+1, j}^{(m)}$, then for $w \in T_{n}^{(m+1)}, h_{u}\left(\tau_{n+1}^{(m+1)}(w)\right)=\tau_{j}^{(m+1)}\left(h_{u}(w)\right)$.

Proof. (1) Assume $u \notin X_{n+1, j}^{(m)}$. We have that $h_{u}\left(p_{n+1}\right)$ and $h_{u}\left(p_{n}\right)$ are both idempotents in $\beta S_{j}^{(m)}$ and $h_{u}\left(p_{n+1}\right) \leqslant h_{u}\left(p_{n}\right)$ because $h_{u}$ is a homomorphism. Assume first that $j<n$ and let $s=u_{1} u_{2} \cdots u_{n}$. Then $s \in \widetilde{S}^{(m)}\left(\begin{array}{c}n \\ j\end{array}\right)$ so $h_{s}\left(p_{n}\right)=p_{j}$ and since $h_{s}$ and $h_{u}$ agree on $S_{n}^{(m)}$, $h_{u}\left(p_{n}\right)=p_{j}$. If $j=n$, then $u=v_{1} v_{2} \cdots v_{n} u_{n+1}$ so $h_{u}$ is the identity on $S_{n}^{(m)}$ and again $h_{u}\left(p_{n}\right)=p_{j}$. Consequently, $h_{u}\left(p_{n+1}\right) \leqslant p_{j}$ and $p_{j}$ is minimal in $\beta S_{j}^{(m)}$ so $h_{u}\left(p_{n+1}\right)=p_{j}$.

(2) It suffices to show that $h_{u}\left(\tau_{n+1}^{(m+1)}(l)\right)=\tau_{j}^{(m+1)}\left(h_{u}(l)\right)$ for each $l \in \mathbb{A}^{(m+1)} \cup$ $\left\{v_{1}, v_{2}, \ldots, v_{n}\right\}$. Now $h_{u}\left(\tau_{n+1}^{(m+1)}\left(x_{m+1}\right)\right)=h_{u}\left(v_{n+1}\right)=u_{n+1}=v_{j}$ and $\tau_{j}^{(m+1)}\left(h_{u}\left(x_{m+1}\right)\right)=$ $\tau_{j}^{(m+1)}\left(x_{m+1}\right)=v_{j}$. If $l \in \mathbb{A}^{(m)}$, then both sides leave $l$ fixed. Finally if $i \in\{1,2, \ldots, n\}$, then $h_{u}\left(\tau_{n+1}^{(m+1)}\left(v_{i}\right)\right)=h_{u}\left(v_{i}\right)=u_{i}$ and $\tau_{j}^{(m+1)}\left(h_{u}\left(v_{i}\right)\right)=\tau_{n+1}^{(m+1)}\left(u_{i}\right)=u_{i}$ because $u_{i} \neq$ $x_{m+1}$.

Lemma 66. Let $q \in E\left(K\left(\beta S_{0}\right)\right)$ and let $r \in E\left(K\left(\beta S_{1}\right)\right)$ such that $r<q$. There is a reductive array $\left\langle p_{i}^{(m)}\right\rangle_{i<2}^{m<\omega}$ of height 2 over $\mathbb{A}$ such that $p_{0}^{(0)}=q$ and $p_{1}^{(0)}=r$.

Proof. Let $p_{0}^{(0)}=q$ and $p_{1}^{(0)}=r$. Let $m \in \mathbb{N}$ and assume that we have chosen $\left\langle p_{i}^{(t)}\right\rangle_{i<2}^{t<m}$ such that for each $t<m$ and each $i \in\{0,1\}, p_{i}^{(t)} \in E\left(K\left(\beta S_{i}^{(t)}\right)\right)$ and $p_{1}^{(t)}<p_{0}^{(t)}$. By Lemma $59, p_{1}^{(m-1)} \in K\left(\beta T_{1}^{(m-1)}\right)$. Since $\tau_{1}^{(m)}$ is an isomorphism from $\beta T_{0}^{(m)}$ onto $\beta T_{1}^{(m-1)}$, 
we may let $p_{0}^{(m)}$ be the unique member of $E\left(K\left(\beta T_{0}^{(m)}\right)\right)$ such that $\tau_{1}^{(m)}\left(p_{0}^{(m)}\right)=p_{1}^{(m-1)}$. By Lemma 60, we may pick $p_{1}^{(m)} \in E\left(K\left(\beta S_{1}^{(m)}\right)\right)$ such that $p_{1}^{(m)}<p_{0}^{(m)}$.

We need to show that for each $u \in \widetilde{S}^{(m)}\left(\begin{array}{l}1 \\ 0\end{array}\right), h_{u}\left(p_{1}^{(m)}\right)=p_{0}^{(m)}$ so let $u \in \widetilde{S}^{(m)}\left(\begin{array}{l}1 \\ 0\end{array}\right)$. Now $p_{0}^{(m)}$ and $p_{1}^{(m)}$ are in $\beta T_{1}^{(m)}, p_{1}^{(m)} \leqslant p_{0}^{(m)}$, and $h_{u}$ is a homomorphism so $h_{u}\left(p_{1}^{(m)}\right) \leqslant h_{u}\left(p_{0}^{(m)}\right)$. Since $h_{u}$ is the identity on $\beta T_{0}^{(m)}, h_{u}\left(p_{0}^{(m)}\right)=p_{0}^{(m)}$ so $h_{u}\left(p_{1}^{(m)}\right) \leqslant p_{0}^{(m)}$. Since $p_{0}^{(m)}$ is minimal in $\beta T_{0}^{(m)}, h_{u}\left(p_{1}^{(m)}\right)=p_{0}^{(m)}$ as required.

Theorem 67. Let $n \in \mathbb{N}$ and assume that $\left\langle p_{i}^{(m)}\right\rangle_{i<n+1}^{m<\omega}$ is a reductive array of height $n+1$ over $\mathbb{A}$. There exist unique $p_{n+1}^{(m)}$ for each $m<\omega$ such that $\left\langle p_{i}^{(m)}\right\rangle_{i<n+2}^{m<\omega}$ is a reductive array of height $n+2$ over $\mathbb{A}$.

Proof. For each $m<\omega$, let $p_{n+1}^{(m)}$ be the identity of the group $G_{n+1}^{(m)}$. This is required by Definition 63(3), so the uniqueness is satisfied. Let $m<\omega$ be given. We need to show that $\left\langle p_{i}^{(m)}\right\rangle_{i<n+2}$ is a reductive sequence over $\mathbb{A}^{(m)}$. Since $G_{n+1}^{(m)} \subseteq p_{n}^{(m)} \beta S_{n+1}^{(m)} \cap \beta S_{n+1}^{(m)} p_{n}^{(m)}$ we have that $p_{n+1}^{(m)} \leqslant p_{n}^{(m)}$. And by Lemma 62 we have that $p_{n+1}^{(m)} \in E\left(K\left(\beta S_{n+1}^{(m)}\right)\right)$.

Now let $0 \leqslant j<i<n+2$ and let $u \in \widetilde{S}^{(m)}\left(\begin{array}{l}i \\ j\end{array}\right)$. We need to show that $h_{u}\left(p_{i}^{(m)}\right)=p_{j}^{(m)}$. If $i<n+1$, this holds by assumption, so assume that $i=n+1$ so that $u \in \widetilde{S}^{(m)}\left(\begin{array}{c}n+1 \\ j\end{array}\right)$. If $j=0$, then $h_{u}$ is the identity on $\beta S_{0}^{(m)}$ so $h_{u}\left(p_{n+1}^{(m)}\right) \leqslant h_{u}\left(p_{0}^{(m)}\right)=p_{0}^{(m)}$ and $h_{u}\left(p_{n+1}^{(m)}\right) \in$ $\beta S_{0}^{(m)}$ so $h_{u}\left(p_{n+1}^{(m)}\right)=p_{0}^{(m)}$. So assume that $j \geqslant 1$. If $u \notin X_{n+1, j}^{(m)}$, then by Lemma 65 , $h_{u}\left(p_{n+1}^{(m)}\right)=p_{j}^{(m)}$.

So we assume that $u=u_{1} u_{2} \cdots u_{n+1} \in X_{n+1, j}^{(m)}$ and let $s=u_{1} u_{2} \cdots u_{n}$. Then we have that $s \in \widetilde{S}^{(m)}\left(\begin{array}{c}n \\ j-1\end{array}\right) \subseteq \widetilde{S}^{(m+1)}\left(\begin{array}{c}n \\ j-1\end{array}\right)$ and hence $h_{u}\left(p_{n}^{(m)}\right)=h_{s}\left(p_{n}^{(m)}\right)=p_{j-1}^{(m)}$ and $h_{u}\left(p_{n}^{(m+1)}\right)=h_{s}\left(p_{n}^{(m+1)}\right)=p_{j-1}^{(m+1)}$.

Combined with Lemma 65, we have that

$$
h_{u}\left(\tau_{n+1}^{(m+1)}\left(p_{n}^{(m+1)}\right)\right)=\tau_{j}^{(m+1)}\left(h_{u}\left(p_{n}^{(m+1)}\right)\right)=\tau_{j}^{(m+1)}\left(p_{j-1}^{(m+1)}\right) .
$$

So as $p_{n+1}^{(m)} \in G_{n+1}^{(m)}=p_{n}^{(m)} \tau_{n+1}^{(m+1)}\left(p_{n}^{(m+1)}\right) \beta S_{n+1}^{(m)} \cap \beta S_{n+1}^{(m)} \tau_{n+1}^{(m+1)}\left(p_{n}^{(m+1)}\right) p_{n}^{(m)}$ we deduce that $h_{u}\left(p_{n+1}^{(m)}\right) \in p_{j-1}^{(m)} \tau_{j}^{(m+1)}\left(p_{j-1}^{(m+1)}\right) \beta S_{j}^{(m)} \cap \beta S_{j}^{(m)} \tau_{j}^{(m+1)}\left(p_{j-1}^{(m+1)}\right) p_{j-1}^{(m)}$. If $j \geqslant 2$, this says that $h_{u}\left(p_{n+1}^{(m)}\right)$ is an idempotent in $G_{j}^{(m)}$ and $p_{j}^{(m)}$ is the identity of $G_{j}^{(m)}$ so $h_{u}\left(p_{n+1}^{(m)}\right)=p_{j}^{(m)}$ as required.

Finally, assume that $j=1$. Then

$$
\begin{aligned}
h_{u}\left(p_{n+1}^{(m)}\right) & \in p_{0}^{(m)} \tau_{1}^{(m+1)}\left(p_{0}^{(m+1)}\right) \beta S_{1}^{(m)} \cap \beta S_{1}^{(m)} \tau_{1}^{(m+1)}\left(p_{0}^{(m+1)}\right) p_{0}^{(m)} \\
& =p_{0}^{(m)} p_{1}^{(m)} \beta S_{1}^{(m)} \cap \beta S_{1}^{(m)} p_{1}^{(m)} p_{0}^{(m)} \\
& =p_{1}^{(m)} \beta S_{1}^{(m)} \cap \beta S_{1}^{(m)} p_{1}^{(m)} .
\end{aligned}
$$

Since $p_{1}^{(m)}$ is minimal in $\beta S_{1}^{(m)}, p_{1}^{(m)} \beta S_{1}^{(m)} \cap \beta S_{1}^{(m)} p_{1}^{(m)}$ is a group with identity $p_{1}^{(m)}$, so $h_{u}\left(p_{n+1}^{(m)}\right)=p_{1}^{(m)}$. 
Combining Lemma 66 and Theorem 67 we obtain:

Corollary 68. For each $p \in E\left(K\left(\beta S_{0}\right)\right)$ there is a reductive array $\left\langle p_{i}^{(m)}\right\rangle_{i<\omega}^{m<\omega}$ of height $\omega$ such that $p_{0}^{(0)}=p$. Morever $p_{1}^{(0)}$ may be taken to be any minimal idempotent of $\beta S_{1}$ such that $p_{1}^{(0)} \leqslant p$.

Corollary 69. Let $p$ be a minimal idempotent in $\beta S_{0}$. There is a sequence $\left\langle p_{n}\right\rangle_{n=0}^{\infty}$ such that

(1) $p_{0}=p$;

(2) for each $n \in \mathbb{N}, p_{n}$ is a minimal idempotent of $\beta \widetilde{S}_{n}$;

(3) for each $n \in \mathbb{N}, p_{n} \leqslant p_{n-1}$;

(4) for each $n \in \mathbb{N}$, each $j \in\{0,1, \ldots, n-1\}$, and each $u \in \widetilde{S}\left(\begin{array}{c}n \\ j\end{array}\right), h_{u}\left(p_{n}\right)=p_{j}$.

Further, $p_{1}$ can be any minimal idempotent of $\beta S_{1}$ such that $p_{1} \leqslant p_{0}$.

Proof. Let $\left\langle p_{i}^{(m)}\right\rangle_{i<\omega}^{m<\omega}$ be as guaranteed by Corollary 68 and for each $i<\omega$ let $p_{i}=p_{i}^{(0)}$. By Lemma 61 each $p_{n} \in E\left(K\left(\beta \widetilde{S}_{n}\right)\right)$.

For several stronger combinatorial consequences of Corollary 69, see Sections 3 and 4 of $[2]$.

To derive the following extension of Theorem 4, we need to restrict to a finite alphabet.

Corollary 70. Assume that $\mathbb{A}$ is finite and for each $m<\omega$, let $\varphi_{m}$ be a finite coloring of $\widetilde{S}_{m}$. For each $m<\omega$, there exists a central subset $C_{m}$ of $\widetilde{S}_{m}$ such that

(1) $\varphi_{m}$ is constant on $C_{m}$ and

(2) whenever $n \in \mathbb{N}$, the set of all $w \in \widetilde{S}_{n}$ such that for each $m<n$, $\left\{w(u): u \in \widetilde{S}\left(\begin{array}{c}n \\ m\end{array}\right)\right\} \subseteq C_{m}$ is central in $\widetilde{S}_{n}$

Proof. Pick $\left\langle p_{m}\right\rangle_{m<\omega}$ as guaranteed by Corollary 69 and for each $m<\omega$ pick $C_{m} \in p_{m}$ with $C_{m} \subseteq \widetilde{S}_{m}$ such that $\varphi_{m}$ is constant on $C_{m}$. Let $n \in \mathbb{N}$ be given. For each $m<n$ and each $u \in \widetilde{S}\left(\begin{array}{c}n \\ m\end{array}\right), h_{u}\left(p_{n}\right)=p_{m}$. Let $D=\bigcap_{m<n} \bigcap\left(h_{u}^{-1}\left[C_{m}\right]: u \in \widetilde{S}\left(\begin{array}{c}n \\ m\end{array}\right)\right)$. Then $D \in p_{n}$. 


\section{References}

[1] V. Bergelson, A. Blass, and N. Hindman, Partition theorems for spaces of variable words, Proc. London Math. Soc. 68 (1994), 449-476.

[2] T. Carlson, N. Hindman, and D. Strauss, An infinitary extension of the GrahamRothschild Parameter Sets Theorem, Trans. Amer. Math. Soc. 358 (2006), 32393262 .

[3] W. Deuber, H. Prömel, B. Rothschild, and B. Voigt, A restricted version of HalesJewett's theorem, Finite and infinite sets, Vol. I, 231-246, Colloq. Math. Soc. János Bolyai 37, North-Holland, Amsterdam, 1984.

[4] R. Graham and B. Rothschild, Ramsey's Theorem for $n$-parameter sets, Trans. Amer. Math. Soc. 159 (1971), 257-292.

[5] A. Hales and R. Jewett, Regularity and positional games, Trans. Amer. Math. Soc. 106 (1963), 222-229.

[6] N. Hindman and D. Strauss, Algebra in the Stone-Čech compactification: theory and applications, second edition, de Gruyter, Berlin, 2012. 